Revue des patrimoines

29 | 2016

Ensembles mobiliers, industriels, techniques.

Connaissance, protection, conservation, présentation au public

\title{
Les collections du Panthéon. Étude, inventaire et perspectives scientifiques
}

\section{Élisabeth Portet}

\section{OpenEdition \\ Journals}

Édition électronique

URL : http://journals.openedition.org/insitu/16152

DOI : 10.4000/insitu. 16152

ISSN : 1630-7305

Éditeur

Ministère de la Culture

Référence électronique

Élisabeth Portet, «Les collections du Panthéon. Étude, inventaire et perspectives scientifiques », In Situ [En ligne], 29 | 2016, mis en ligne le 27 juillet 2018, consulté le 10 décembre 2020. URL : http:// journals.openedition.org/insitu/16152 ; DOI : https://doi.org/10.4000/insitu.16152

Ce document a été généré automatiquement le 10 décembre 2020.

\section{(c) (1) $(9)$}

In Situ Revues des patrimoines est mis à disposition selon les termes de la licence Creative Commons Attribution - Pas d'Utilisation Commerciale - Pas de Modification 4.0 International. 


\title{
Les collections du Panthéon. Étude, inventaire et perspectives scientifiques
}

\author{
Élisabeth Portet
}

Rarement un édifice n'aura autant changé d'affectation au gré des soubresauts de l'histoire que le Panthéon (fig. 1). L'historien Edgar Quinet résume cette destinée complexe en 1883 : «Monument de Janus au double visage, l'un tourné vers le passé, l'autre vers l'avenir, il change de nom suivant la différence des temps! Regardez! Église ou temple, Sainte-Geneviève ou Panthéon, il pourrait à lui seul dire si la Révolution est vaincue ou victorieuse ${ }^{1}$. » Tout au long du $\mathrm{XIX}^{\mathrm{e}}$ siècle, le Panthéon oscille entre un usage religieux et un usage laïque. 
Figure 1

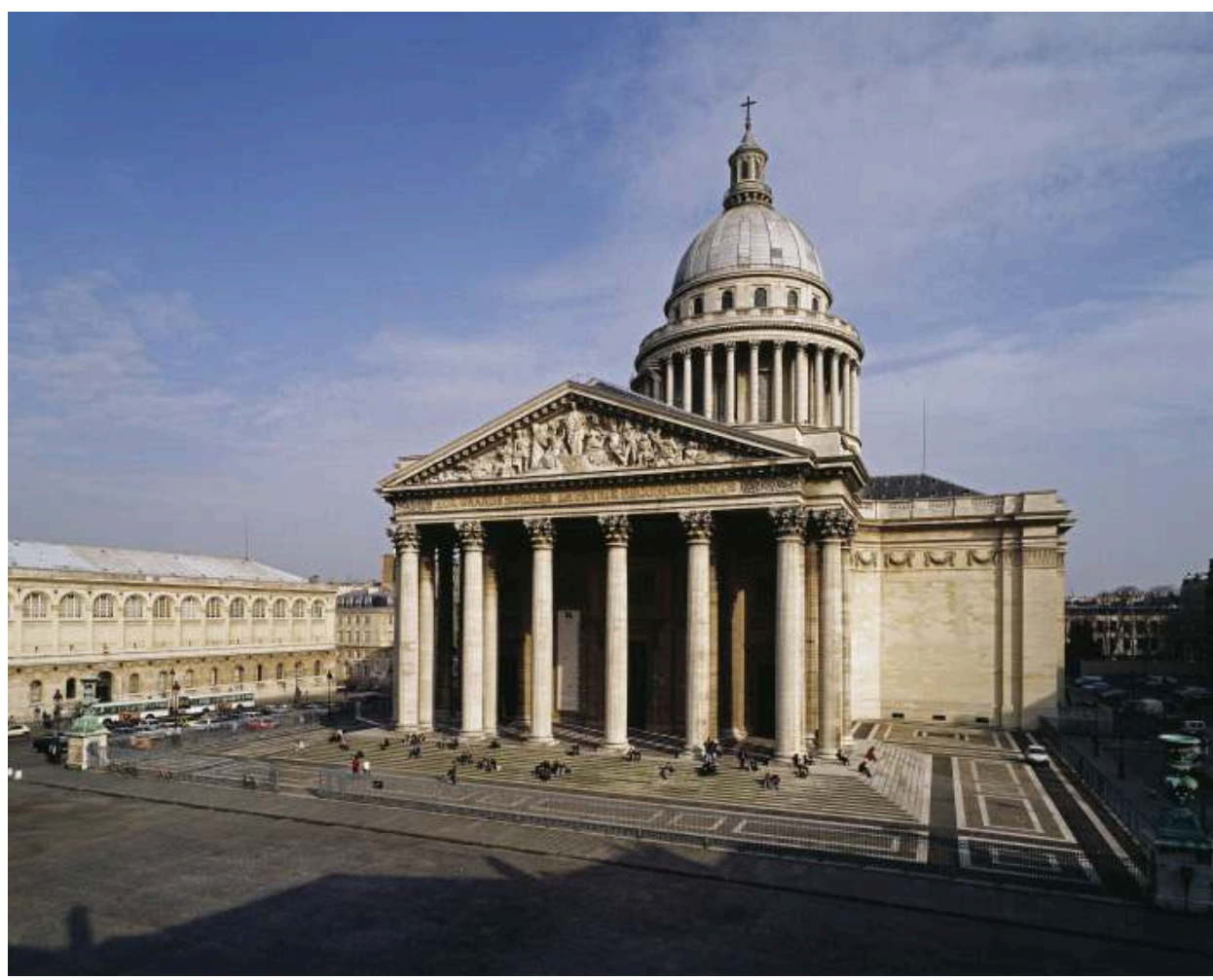

Le Panthéon, façade principale dans l'axe de la rue Soufflot.

Phot. Jean-Luc Paillé. (c) Jean-Luc Paillé / Centre des monuments nationaux, 2000.

2 L'ancienne église Sainte-Geneviève, élevée par Jacques-Germain Soufflot (1713-1780) à la demande de Louis $\mathrm{XV}^{2}$, est transformée en lieu d'inhumation des grands hommes de la Nation pendant la Révolution, en 1791. Ainsi modifiée en temple républicain, elle est rendue au culte catholique avec le retour des Bourbons au pouvoir, en 1816. Redevenant temple civique sous la monarchie de Juillet puis à nouveau église sous le Second Empire, elle voit finalement sa vocation républicaine et mémorielle consacrée dans un décret du 26 mai 1885, quelques jours après le décès de Victor Hugo, qui rend au Panthéon sa « destination primitive et légale. Les restes des grands hommes qui ont mérité la reconnaissance nationale y seront déposés $»^{3}$. Il ne se départit plus de cette affectation, qui perdure aujourd'hui à travers la diffusion des valeurs universelles ${ }^{4}$, récemment renforcées lors des dernières cérémonies de " panthéonisation » en $2015^{5}$ et en $2018^{6}$.

3 Ces changements ont favorisé le développement de programmes décoratifs ambitieux qui constituent le cœur des collections du Panthéon. Cependant, la rapidité des transformations de l'édifice a parfois laissé les projets à l'état d'esquisse ou entraîné des modifications en profondeur, quand elle n'a pas causé la destruction des œuvres en place lorsqu'elles n'étaient plus en adéquation avec le décor souhaité. Ces revirements ne facilitent guère la compréhension des collections qui ne résultent pas d'un programme unique mais sont les vestiges disparates des remaniements successifs de l'édifice.

4 Dans ce cas particulier, où les collections se révèlent indissociables de leur environnement architectural initial, le travail d'inventaire s'est attaché à réunir les ressources documentaires disponibles et à approfondir les recherches sur des 
ensembles mobiliers jusqu'alors méconnus. Replacer chaque bien culturel dans son contexte a été le fil conducteur de ce travail qui s'est déroulé entre 2005 et 2016.

\section{Le chantier de l'inventaire et du récolement}

Dans le cadre des missions de conservation, de restauration et d'entretien des monuments et des collections appartenant à l'État qui lui sont remis en dotation, le Centre des monuments nationaux a mis en place des instruments de connaissance pour améliorer la gestion et l'étude de ses collections. Dans cette perspective, la réalisation des inventaires et le pilotage des campagnes de récolement au sein d'un service dédié, créé en 2005, ont permis leur valorisation auprès du public.

De la phase d'enquête in situ à la diffusion des données, la méthode appliquée aux opérations d'inventaire et de récolement suit un protocole spécifique de classement et de normalisation qui vise à l'harmonisation des résultats sur l'ensemble du réseau géré par l'établissement ${ }^{7}$. À l'exception de l'étude thématique transversale sur les tapisseries lancée en 2007 - dont les travaux ont été publiés en ligne en $2017^{8}$ - le recensement des collections privilégie une approche par monument afin de pouvoir disposer progressivement d'un inventaire exhaustif pour chacun des 76 sites qui conservent des collections, soit près de 116300 biens culturels estimés à ce jour. Cette mission est conduite dans un souci permanent de cohérence des ensembles mobiliers. Pour le Panthéon, ce travail totalise 637 biens culturels portés à l'inventaire des collections. Il offre désormais une vision complète de l'intégralité des objets mobiliers conservés dans le monument (fig. 2). 
Figure 2

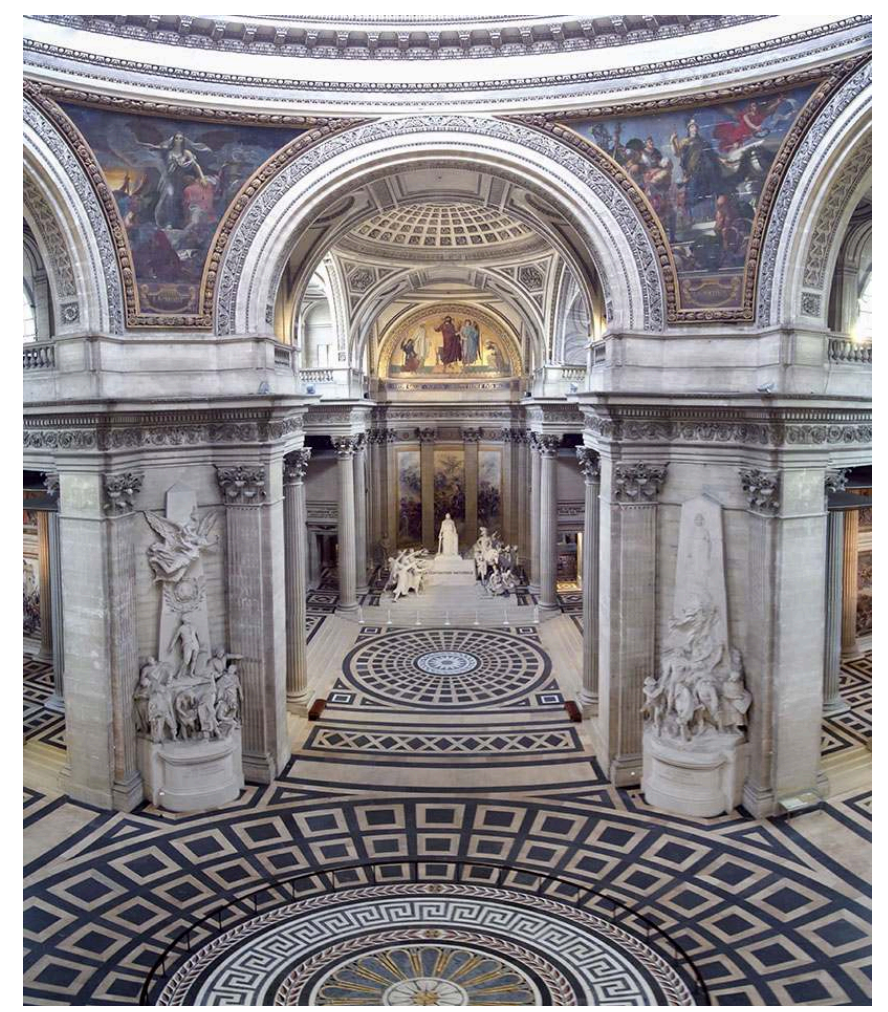

Vue intérieure du Panthéon en direction du chœur.

Phot. Christian Gluckman. (C) Christian Gluckman / Centre des monuments nationaux, 2016.

\section{Recherches et documentation}

7 La première étape consiste à élaborer un dossier documentaire complet pour rassembler les sources disponibles et collecter les inventaires existants. La bibliographie de référence offre un panorama détaillé du contexte de construction de l'église initiale, de ses transformations et de ses décors.

8 À cet égard, il est intéressant de remarquer que le Panthéon fait partie du vaste projet d'inventaire initié par la Troisième République pour répertorier les monuments emblématiques du patrimoine français à travers son Inventaire des richesses d'art de la France $^{9}$. Les décors mentionnés dans cet ouvrage sont précisément décrits et apportent des informations sur l'état historique de l'édifice en 1889. Cette ressource incontournable a été complétée par la consultation du catalogue de l'exposition « Le Panthéon. Symbole des révolutions $»^{10}$ qui s'est tenue à Paris et à Montréal en 1989. Cette remarquable synthèse dresse un état très complet de la recherche menée à l'occasion des célébrations du bicentenaire de la Révolution.

9 La plupart des œuvres ayant fait l'objet d'une commande de l'État, les sources sont conservées et consultables aux Archives nationales. Les séries F13 (édifices publics et travaux urbains de la Révolution à la monarchie de Juillet) et F21 (dossiers de l'administration des Beaux-Arts) permettent de suivre la genèse des programmes de travaux au XIX ${ }^{\mathrm{e}}$ siècle. Les anciens rapports et les mémoires des projets ainsi que les études préalables menées ces dernières années ont été consultés aux archives de la Médiathèque de l'architecture et du patrimoine pour appréhender les campagnes de 
restauration qui ont porté aussi bien sur le bâti que sur les collections. Cette documentation a été croisée avec la recherche de vues anciennes des intérieurs de l'édifice et des célébrations nationales.

\section{Définition du corpus}

10 La prise en compte des objets mobiliers inscrits à l'inventaire nécessite de définir des critères de sélection dont les orientations sont sans cesse en évolution. Dans une logique administrative et gestionnaire, le corpus concerné par l'inventaire du Panthéon s'est concentré sur l'ensemble des biens meubles. Les décors scellés et encastrés dans les maçonneries, les revêtements de sols et de murs n'ont pas été inscrits à l'inventaire des collections en raison de leur statut d'immeuble par nature et d'immeuble par destination.

11 Toutefois, le cas spécifique du Panthéon, où les décors sont compris dans les collections, a entraîné des adaptations pour intégrer certaines catégories de biens culturels. Par exemple, les toiles marouflées (fig. 3) qui recouvrent les murs des quatre nefs ont été portées à l'inventaire en raison de leur prise en charge spécifique par les équipes de l'établissement en vue de leur restauration au titre des collections. Un des enjeux de ce travail est d'ailleurs de fournir un support de programmation des futures interventions grâce aux constats d'état réalisés. Ces derniers, même sommaires, permettent de cibler les besoins et de dégager les priorités en matière de conservationrestauration.

Figure 3

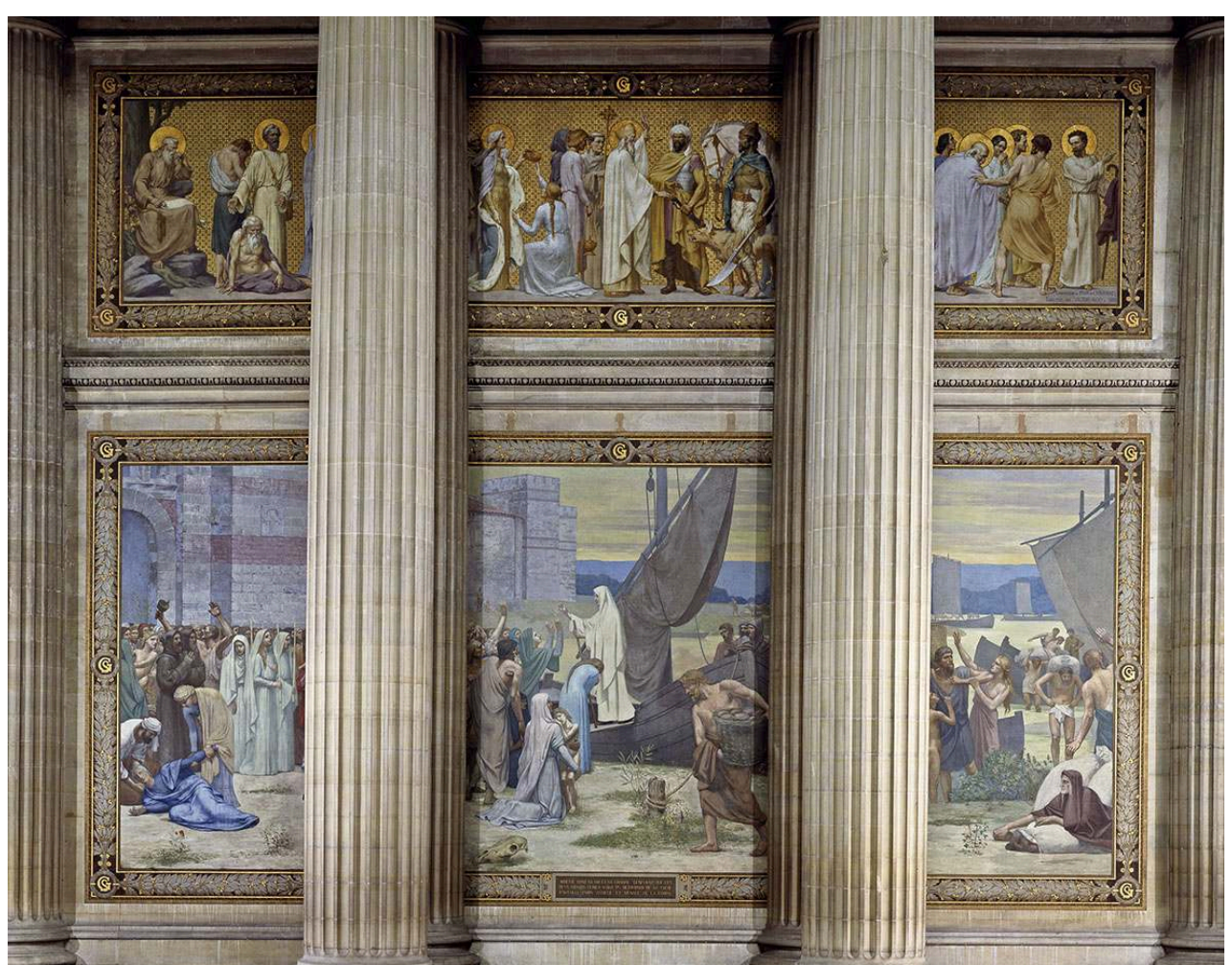

Pierre Puvis de Chavannes, Sainte Geneviève ravitaillant Paris assiégé, 1898.

Phot. Caroline Rose. (c) Caroline Rose / Centre des monuments nationaux, 1991. 
L'extension constante du champ patrimonial, le dialogue permanent des objets mobiliers avec leur environnement architectural et les enjeux de leur conservation matérielle ont contribué à dresser l'inventaire de la collection d'estampages réalisés sur le relief du fronton de David d'Angers lors des travaux de restauration entrepris de 1967 à 1969 par le sculpteur Carlo Sarrabezolles (1888-1971) (fig. 4).

Figure 4

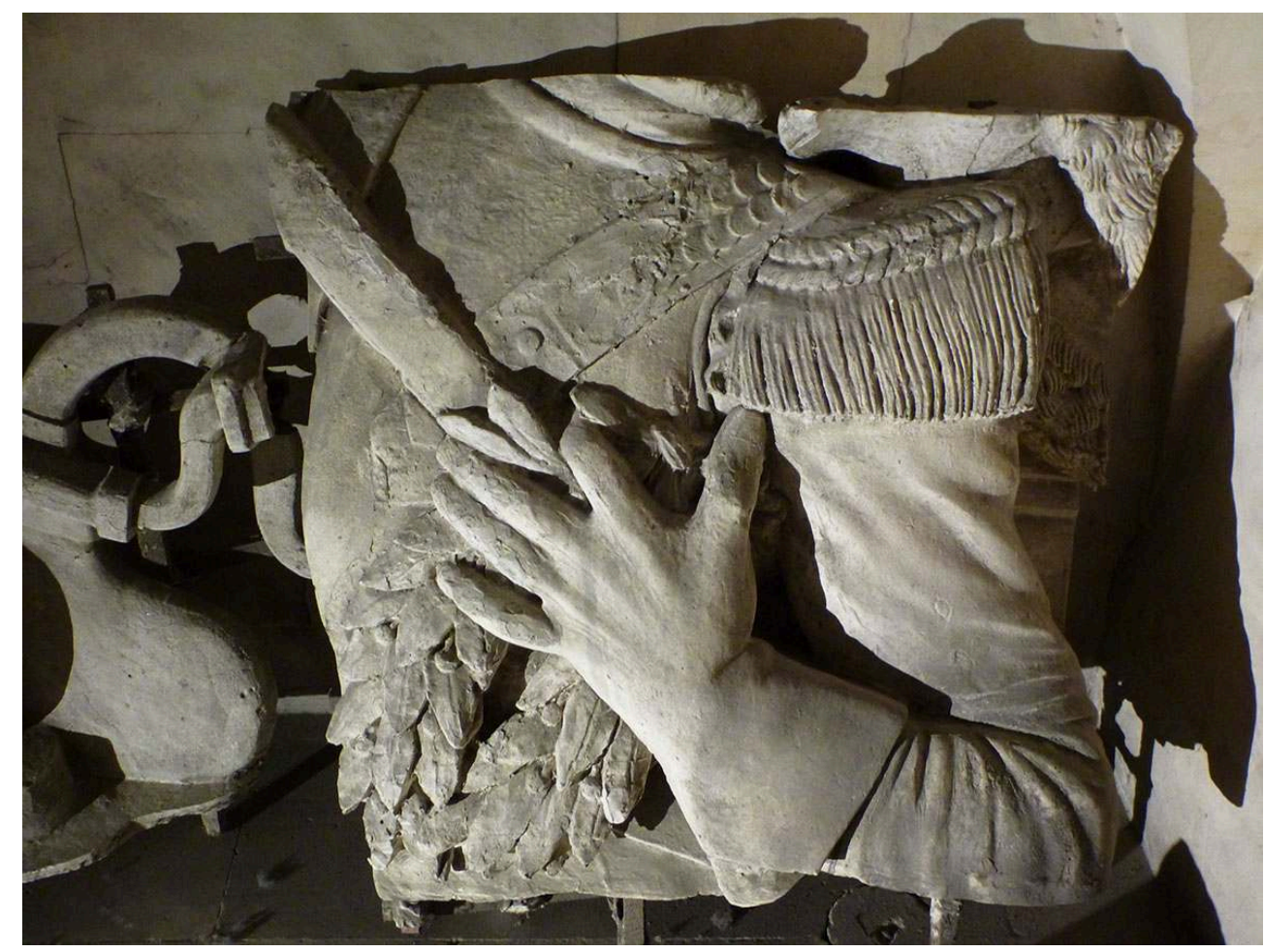

TORSE, ESTAMPAgE DE CARLO SARRABEZOLLES.

Phot. Élisabeth Portet. @ Élisabeth Portet / Centre des monuments nationaux, 2012.

13 Dans une même perspective de sauvegarde, le moulage du chat égyptien d'après l'original du musée du Louvre, exécuté en 1996 à l'occasion de la cérémonie de " panthéonisation » d'André Malraux, a été consigné dans cet inventaire.

\section{Organisation de la chaîne patrimoniale}

Une fois le champ d'étude défini, les enquêtes sur site se sont déroulées en plusieurs phases, à partir de 2005, selon un rythme pluriannuel. Pour faciliter le repérage, le travail a d'abord porté sur les collections présentées dans le parcours de visite avant de se concentrer sur celles qui ne sont pas visibles du public ${ }^{11}$. Cette première approche par emplacement a été suivie d'un examen approfondi par catégorie d'objets afin d'identifier les matériaux constitutifs des œuvres et les techniques employées. Ce recensement a souligné la nécessité d'élaborer un protocole d'échantillonnage pour la prise à l'inventaire des ornements funéraires de la crypte. L'opération, effectuée en collaboration avec la direction régionale des Affaires culturelles (DRAC) d'île-de-France, a permis d'entreprendre leur sauvegarde et de programmer leur prise en charge en conservation préventive. 

nécessaire pour mener à bien l'inventaire du lapidaire conservé dans les tribunes (fig. 5).

Figure 5

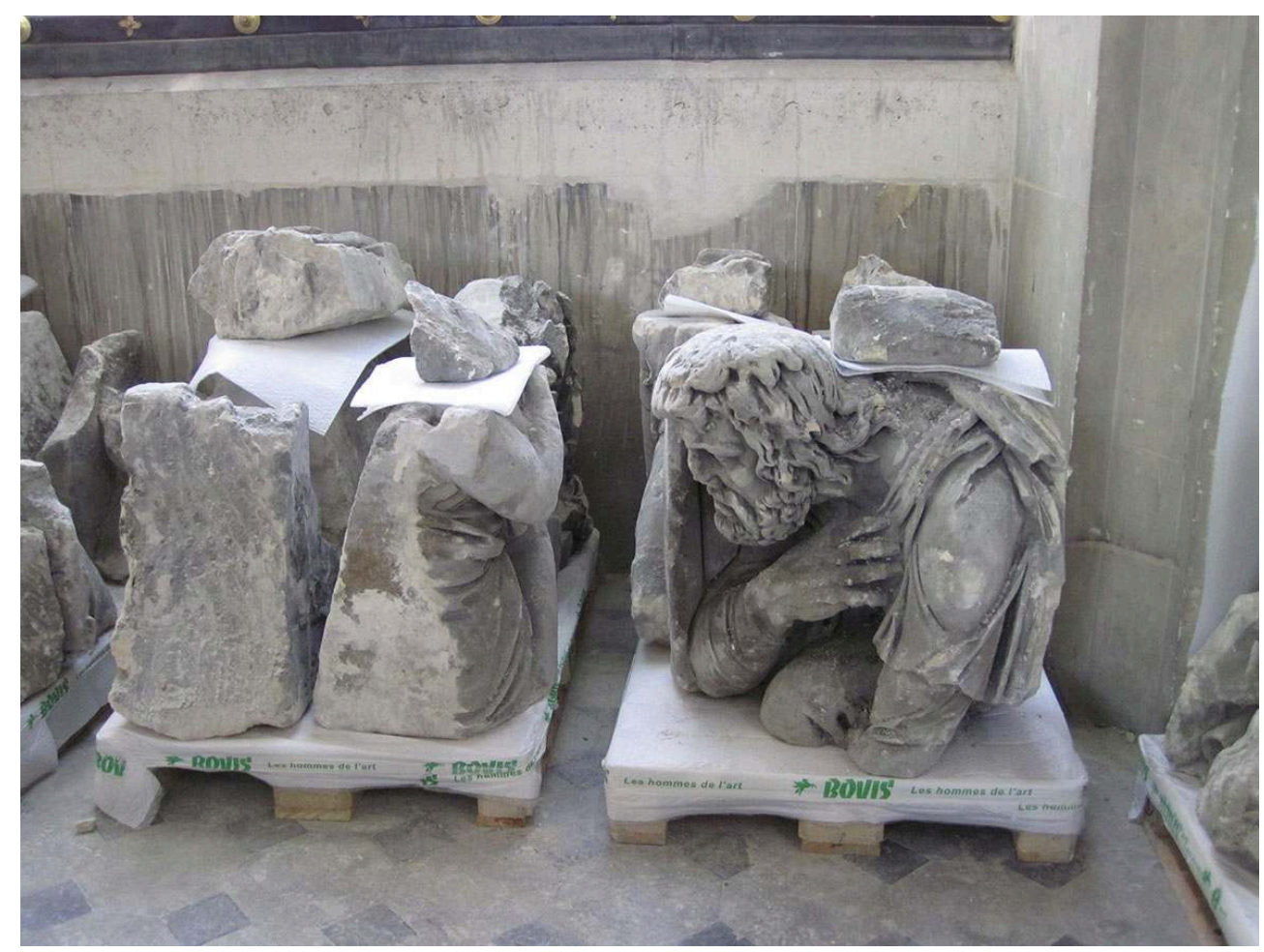

Lapidaire des tribunes après inventaire et rangement sur palettes.

Phot. Francine Pin. (c) Francine Pin / Centre des monuments nationaux, 2015

Chaque bien culturel inscrit à l'inventaire fait l'objet d'une notice comprenant une description normée à partir du système descriptif des objets mobiliers et du thésaurus Palissy ${ }^{12}$, accompagnée d'un visuel et d'un commentaire historique. Le relevé des inscriptions et des marquages anciens est systématiquement effectué. La synthèse de ces informations est ensuite reportée sur la base de données d'inventaire et de gestion des collections du Centre des monuments nationaux ${ }^{13}$. Cet outil informatisé, nommé Collectio, permet d'interroger les différents champs de la base selon des modes de requête spécifiques, en attendant la mise en place d'une interface de consultation en ligne pour le public. Cette étape indispensable attribue un numéro à chaque objet qui est composé d'un trigramme comprenant les trois premières lettres du monument (PAN), suivies de la date d'entrée du bien dans les collections et d'un numéro séquentiel à six chiffres. Lorsque les dates d'entrée ne sont pas connues, c'est l'année d'enquête qui est retenue.

17 La vérification de la protection des objets mobiliers au titre des monuments historiques est effectuée à ce stade pour indiquer cette mention sur chaque notice d'inventaire. Les collections du Panthéon bénéficient d'une large couverture de classement, à hauteur de $41 \%$. Dans une volonté de préservation, la maquette en pierre de Jean-Baptiste Rondelet (voir fig. 12) ou le calorifère de Louis-Pierre Baltard (fig. 6) ont fait l'objet d'une protection en tant qu'objets, respectivement en 1975 et 1977. Ces mesures ont été 
étendues en 1991 avec le classement des plâtres, témoins des décors originaux disparus ${ }^{14}$. Le dépôt lapidaire, conservé dans les tribunes, avait été classé avec le bâti en 1920. Il faut rappeler qu'à cette époque, le Panthéon faisait partie des monuments du $\mathrm{XVIII}^{\mathrm{e}}$ siècle nouvellement pris en compte parmi les édifices protégés.

Figure 6

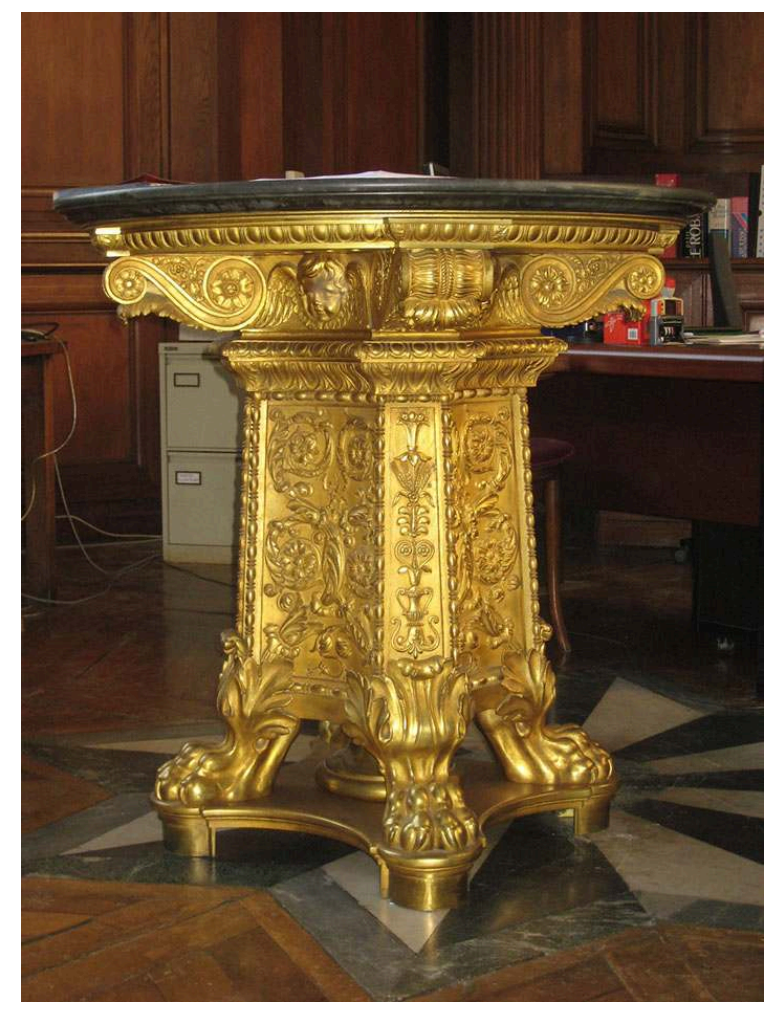

Calorifère en bronze doré de Baltard classé au titre des monuments historiques.

Phot. Élisabeth Portet. ( ) Élisabeth Portet / Centre des monuments nationaux, 2007.

\section{Identification et gestion des dépôts}

Parallèlement, la situation juridique de chaque bien culturel a été clarifiée afin de préciser l'historique des provenances. Cette opération vise notamment à identifier les biens mis en dépôt et à assurer leur suivi administratif en lien avec la Commission de récolement des dépôts d'œuvres d'art de l'État ${ }^{15}$.

Le statut particulier des collections du Panthéon mérite d'être souligné car pour l'essentiel, elles s'inscrivent dans le cadre de la commande publique. À ce titre, leur gestion relève du Centre national des arts plastiques (CNAP), même si elles ne sont pas portées sur ses inventaires. Un transfert de gestion pour simplifier la politique des dépôts d'œuvres d'art de l'État est envisagé dans un calendrier dont l'échéance et les modalités restent à définir.

À cet ensemble majoritaire, il faut ajouter les deux grandes pièces murales (tapisseries), commandées en 1875 pour le monument alors redevenu église et tissées aux Gobelins, qui sont inscrites sur les inventaires du Mobilier national. Si le Centre des monuments nationaux est dépositaire de ces biens, il a initié également une politique de dépôts à l'extérieur afin de mettre en valeur les collections non exposées. 
La maquette en plâtre à demi-grandeur de la fontaine de Noyon par François Masson (1745-1807) s'inscrit ainsi dans cette démarche et fait l'objet d'un dépôt au musée du Noyonnais (Noyon, Oise) depuis 2003. Le récolement des dépôts est aussi l'occasion d'effectuer des découvertes. Le plâtre original de la statue du général Hoche par Jules Dalou (1838-1902) (fig. 7) a été retrouvé en 2006 dans un entrepôt municipal de la Ville de Versailles où l'œuvre avait été déposée près de vingt ans auparavant pour une exposition à l'hôtel de ville. Son retour dans le monument a été organisé en 2008 à son dernier emplacement connu, à proximité du chœur ${ }^{16}$.

\section{Figure 7}

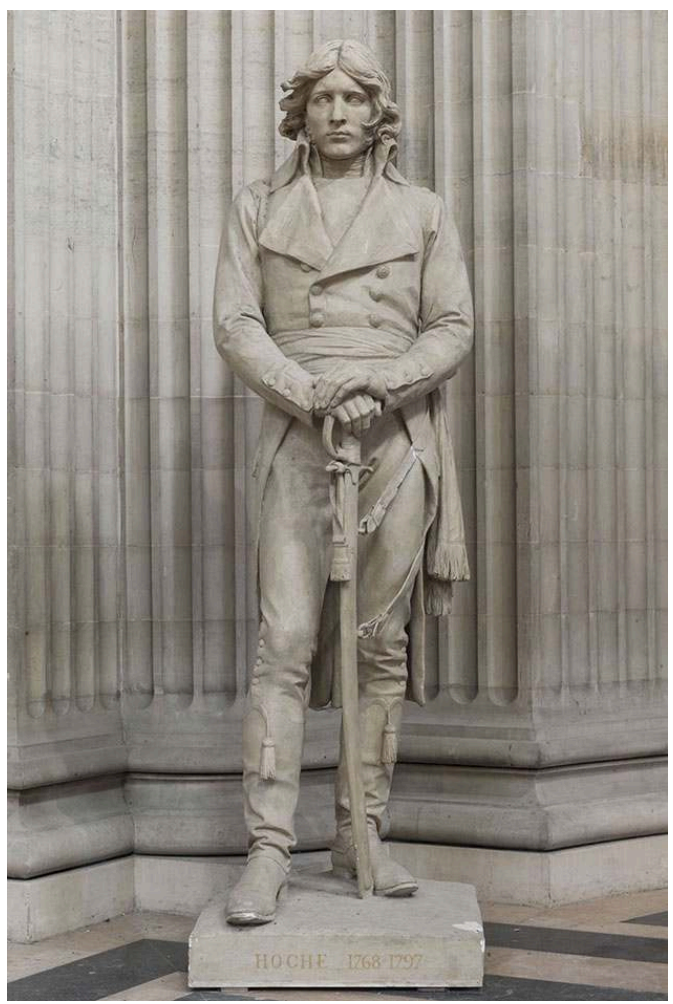

Plâtre de la statue du général Hoche par Jules Dalou.

Phot. Benjamin Gavaudo. (C) Benjamin Gavaudo / Centre des monuments nationaux, 2016.

\section{Caractéristiques générales}

22 Les collections du Panthéon sont essentiellement composées de peintures et de sculptures (fig. 8). Leur répartition fait apparaître deux grands ensembles. Le premier regroupe les commandes de l'État aux artistes chargés d'exécuter des décors à portée didactique; il est prépondérant puisqu'il s'agit de montrer des œuvres en parfaite adéquation avec l'usage du monument. Pour cette raison, une partie des collections comprend des fragments de décors d'architecture restés à l'état d'esquisses et déposés au gré des remaniements de l'édifice. Le second ensemble est constitué par des acquisitions qui distinguent deux catégories: les hommages funèbres et les achats effectués par l'établissement entre 2003 et 2012. 


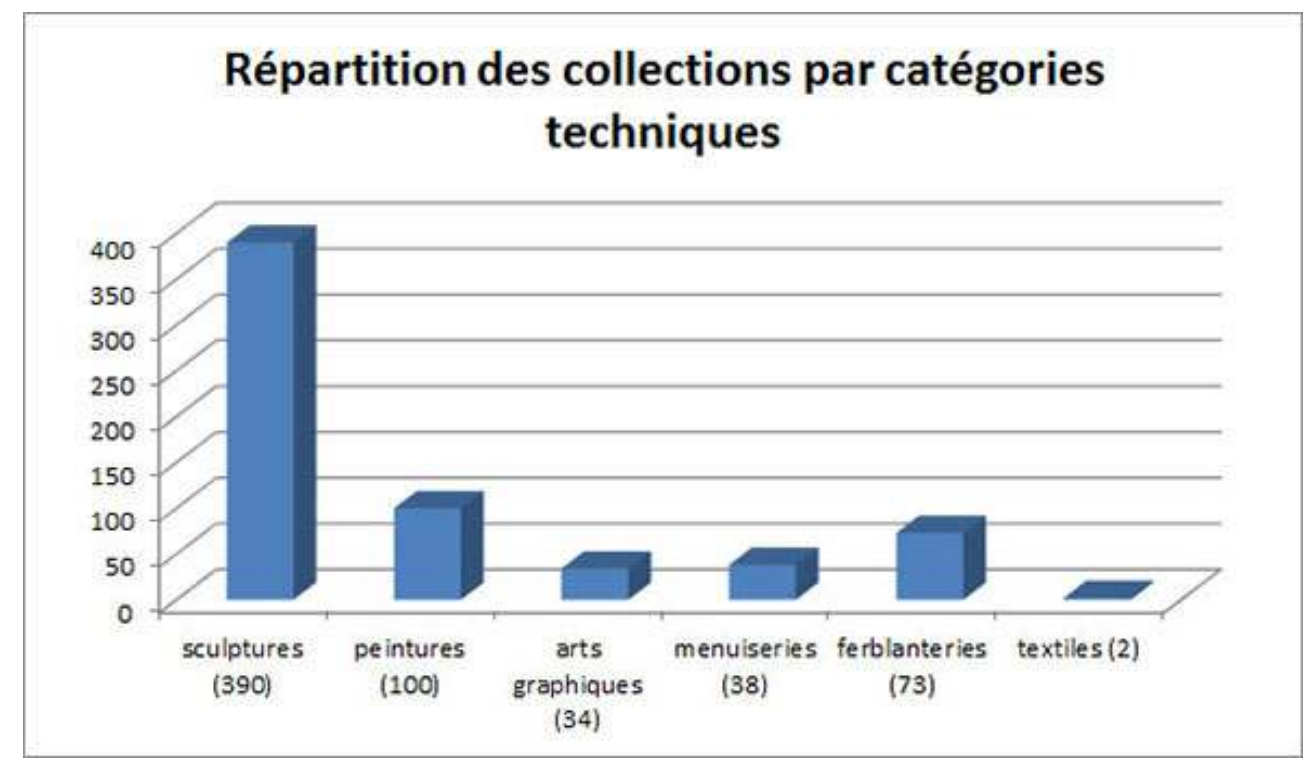

Répartition des collections.

Repro. Élisabeth Portet. (C Élisabeth Portet / Centre des monuments nationaux, 2015.

\section{Les ensembles mobiliers à l'épreuve du temps}

Évoquer les collections du Panthéon, c'est se pencher sur la coexistence de nombreux décors, parfois incompatibles, qui illustrent la volonté de chaque régime politique d'écrire l'histoire. Leur étude fait apparaître tous les stades de la création, de l'esquisse - peinte ou sculptée - à l'œuvre définitive, de l'œuvre en place - originale ou restituée à l'œuvre déposée, et toutes les techniques : taille de pierre, moulage, peinture à l'huile et à la cire, menuiserie, ferronnerie, orfèvrerie ou tapisserie.

Des décors de l'église Sainte-Geneviève aux grandes commandes de la Troisième République en passant par les hommages funèbres, la richesse des collections du Panthéon permet de suivre l'évolution des différents états historiques. Pour plus de clarté, leur présentation développée ci-après privilégie un cheminement chronologique.

\section{Les décors de l'église Sainte-Geneviève}

De l'église en forme de croix grecque surmontée d'un dôme construite par Soufflot à partir de 1764 et dédiée à sainte Geneviève selon le vœu royal ${ }^{17}$, il reste quelques fragments en plâtre à demi-grandeur d'exécution réalisés à partir de 1776 pour l'ornementation sculptée du péristyle et le décor voûté des quatre nefs. Ces œuvres ont valeur de témoignage puisque les reliefs originaux ont été supprimés lors des premières transformations de l'édifice, devenu Panthéon. Cette collection de plâtres issus de moules à pièces, comme le montrent les traces de coutures sur la surface, révèle le processus créatif et permet de suivre les étapes de réalisation du décor architectural. Ils ont été identifiés lors d'un travail d'inventaire mené en 1988-1989 ${ }^{18}$ à l'occasion de travaux de restauration dans les combles du Panthéon. Cette importante découverte a lancé la démarche de regroupement des fragments par grands ensembles 
en fonction de leur provenance (fig. 9). Le récolement mené en 2010 s'est donc attaché à vérifier la présence des éléments répertoriés et à reporter les données actualisées sur l'inventaire informatisé des collections.

Figure 9

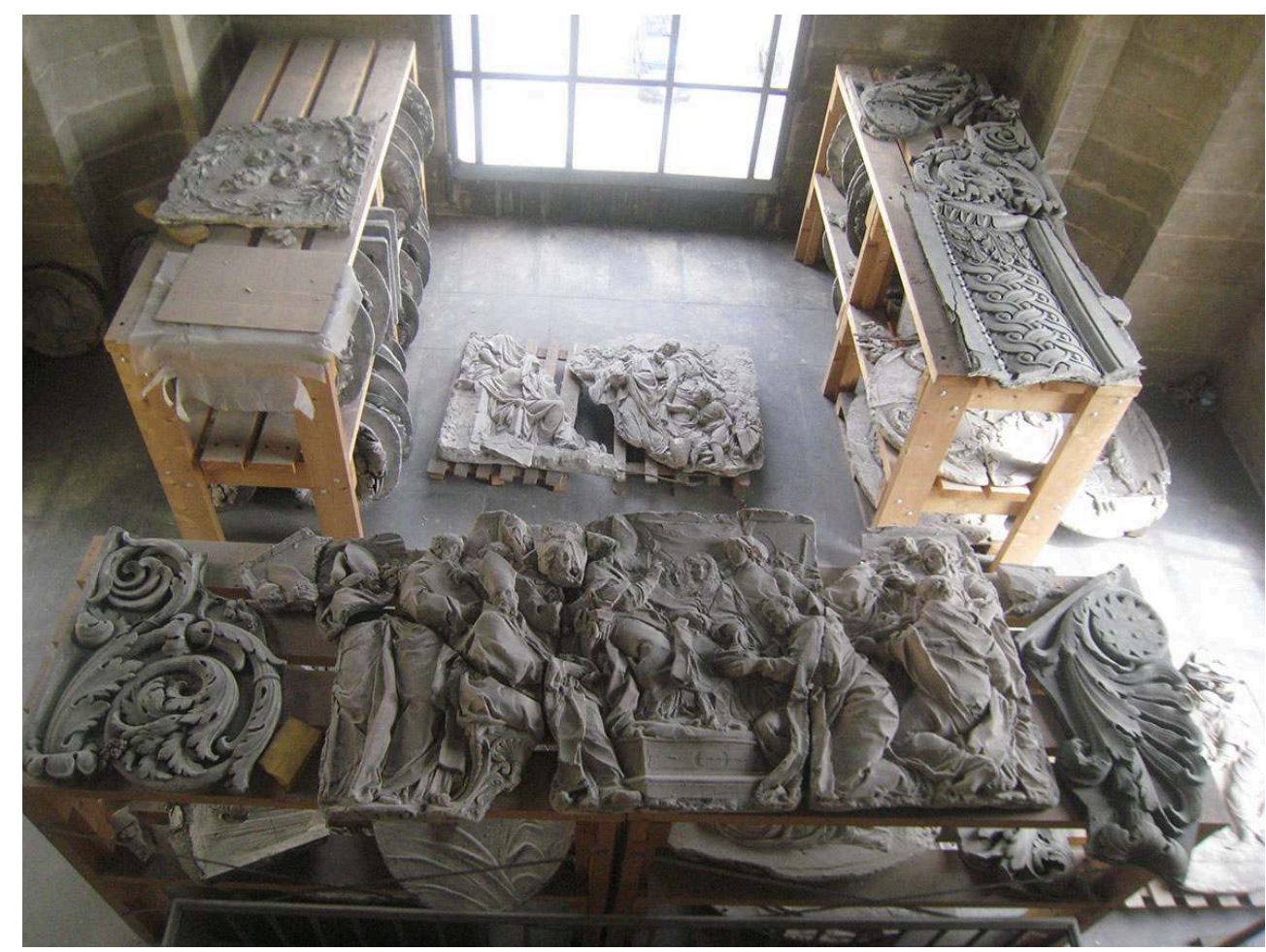

Dépôt des plâtres dans la tour nord-est.

Phot. Élisabeth Portet. ( E Élisabeth Portet / Centre des monuments nationaux, 2010.

Destinés à glorifier la religion chrétienne, les premiers groupes de fragments appartiennent au décor extérieur du péristyle dont les plâtres de deux bas-reliefs parmi les cinq anciennement en place sont partiellement conservés. Au moins dix-neuf éléments sont attribués au relief central montrant Sainte Geneviève distribuant du pain aux pauvres dans un temps de famine par Jacques-Philippe de Beauvais (1739-1781). Un autre tirage, brisé et lacunaire, appartient au relief de droite, Sainte Geneviève rendant la vue à sa mère par Pierre Julien (1731-1804). Le deuxième groupe est constitué de fragments provenant des décors intérieurs des quatre nefs dont l'iconographie est consacrée à l'Ancien Testament (ouest), à l'Église grecque (nord), à l'Église romaine (sud) et à l'Église gallicane (est). Hormis la nef sud, les plâtres subsistants offrent un aperçu des différents décors en place. Dans la nef ouest, les pendentifs figurant Moïse, Aaron, David et Josué étaient accompagnés de médaillons ovales montrant des épisodes de la vie de ces patriarches par Julien, Beauvais et Dupré ${ }^{19}$. Six médaillons sont identifiés : Isaac bénissant Jacob (fig. 10), Consécration d'Aaron, Présentation du Temple au roi Salomon, Le Serpent d'airain, Le Sacrifice d'Isaac et la Consécration d'un autel, qui permettent d'apprécier la finesse d'exécution du relief à travers le traitement des personnages et le travail des draperies. Ces motifs étaient accompagnés d'un triangle équilatéral environné de rayons et de nuages encore en place aujourd'hui. Dans la nef est, seule la moitié du programme fut réalisée, mais il reste deux médaillons en plâtre identifiés: Saint Césaire enfant donne ses habits aux pauvres et Saint Polycarpe enseigne à 
Irénée enfant. Dans la nef nord, le modèle en plâtre du labarum de Constantin qui figurait au centre de la voûte a été retrouvé. Les descriptions mentionnées dans les mémoires de l'époque ${ }^{20}$, complétées par l'observation des gravures anciennes et du bâti actuel, ont permis d'effectuer les rapprochements nécessaires pour définir, dans la mesure du possible, l'appartenance de chaque fragment à son cadre architectural initial. Le programme iconographique, défini par Soufflot avec le sculpteur Guillaume II Coustou (1716-1777), devait affirmer la perpétuité de la religion chrétienne et de la monarchie. Il faut rappeler que l'édifice est bâti suivant la volonté royale à l'emplacement de l'église initiale construite par Clovis sur le lieu de la sépulture de la patronne de Paris. Les sculpteurs sollicités sont essentiellement des élèves de Coustou et des anciens pensionnaires à l'Académie de France à Rome qui bénéficient, pour la plupart, de leur première commande officielle.

Figure 10

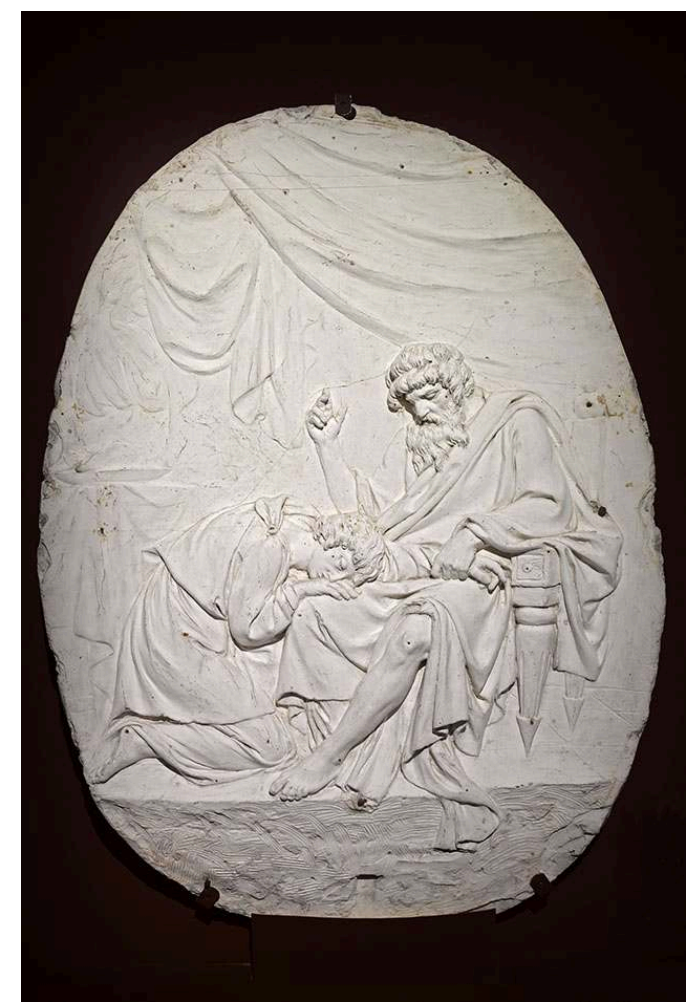

Pierre Julien, Isaac bénissant Jacob, médaillon en plâtre, vers 1776-1780.

Phot. Patrick Cadet. (C) Patrick Cadet / Centre des monuments nationaux, 2013.

\section{Les collections du Panthéon de la Révolution}

En 1791, les transformations de l'édifice - l'église n'avait pas encore été consacrée - en lieu d'inhumation des grands hommes nécessitent des aménagements spécifiques qui s'accompagnent d'un renouvellement du décor en place ${ }^{21}$. De cette période, est conservée une partie des décors du péristyle. Un plâtre à demi-grandeur d'exécution a été identifié comme appartenant au bas-relief central de Guillaume Boichot (1735-1814) La Nature offrant à la France la Déclaration des droits de l'homme (fig. 11). Les originaux en pierre de ce nouveau programme défini par l'architecte Antoine Quatremère de Quincy (1755-1849) existent à l'état fragmentaire en raison de leur dépose lors des 
remaniements suivants. Au moins trois éléments lapidaires, la corne d'abondance et les têtes féminines de la France et de la Nature, se rapportent au relief central de Boichot. Les autres blocs taillés appartiennent aux reliefs latéraux du péristyle, L'Empire de la loi par Auguste-Félix Fortin (1763-1832) et la Nouvelle Jurisprudence par Pierre-Laurent Roland (1746-1816). Bien que lacunaires, ces exemples permettent d'appréhender l'échelle monumentale de ces sculptures programmatiques.

Figure 11

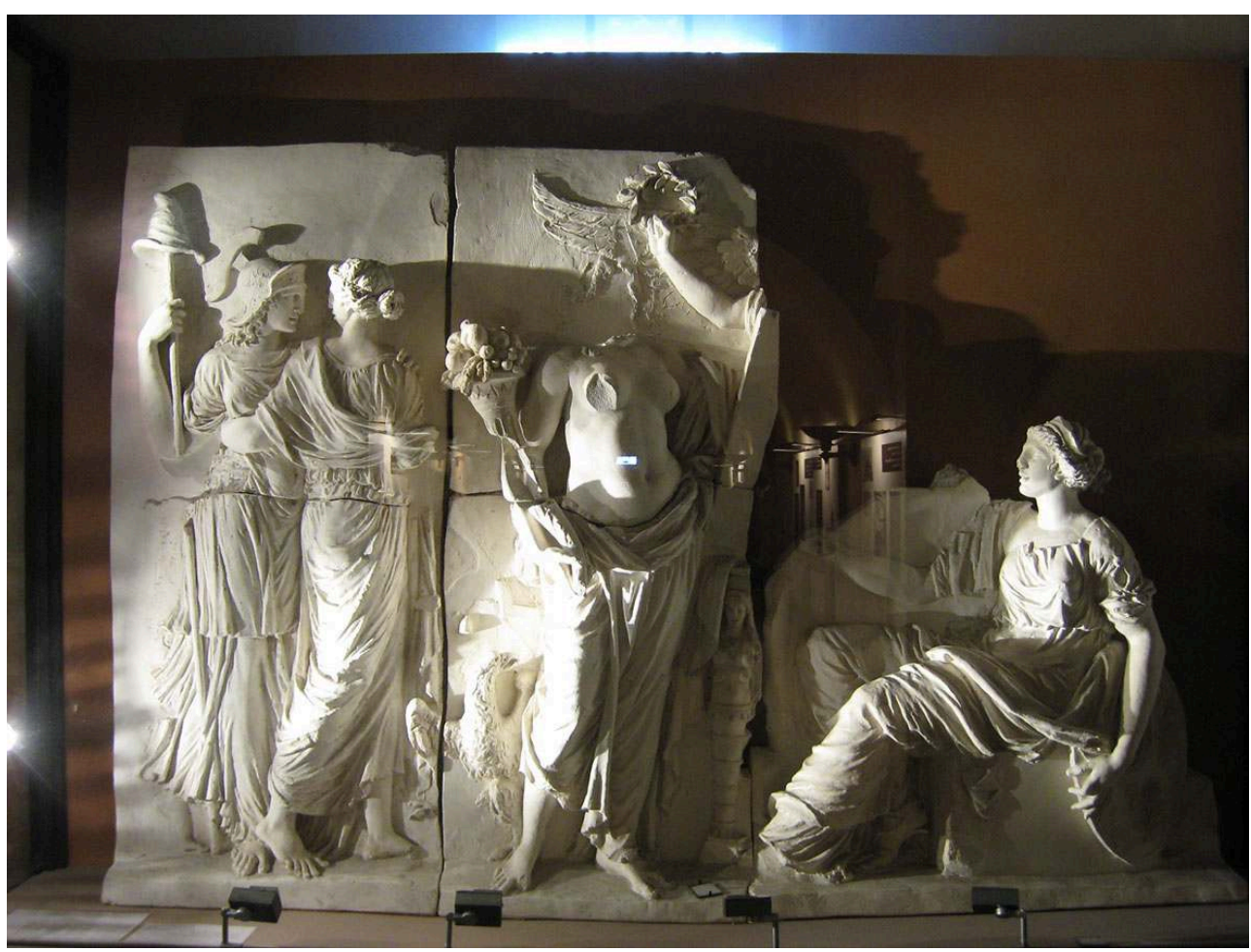

Guillaume Boichot, La Nature offrant à la France la Déclaration des droits de l'homme, relief en plâtre, 1793.

Phot. Élisabeth Portet. (c) Élisabeth Portet / Centre des monuments nationaux, 2008.

Les changements n'affectent pas que les décors, ils concernent aussi l'architecture, dont les modifications apparaissent sur la maquette polychrome en pierre et en plâtre (fig. 12) probablement exécutée par Jean-Baptiste Rondelet (1743-1829), élève de Soufflot qui poursuit la réalisation du gros œuvre après 1780 . Si les clochers de l'église sont encore présents, les fenêtres basses ont déjà été murées et le lanternon modifié pour correspondre au nouvel usage laïque. Contemporaine de la construction de l'édifice et retouchée au fur et à mesure de l'évolution du chantier, la maquette présentait le monument au public dans une démarche pédagogique novatrice pour l'époque. Sa présence est attestée dans le monument dès $1773^{22}$, et un balcon avait été aménagé pour les visiteurs venus la contempler. Une partie traitée en écorché montre le traitement des trois coupoles superposées à l'intérieur du dôme qui suscitèrent de vives controverses sur leur capacité de portance. Devant le défi technique d'un tel ouvrage, les contemporains de Soufflot avaient émis de grandes inquiétudes sur la stabilité de l'édifice. 
Figure 12

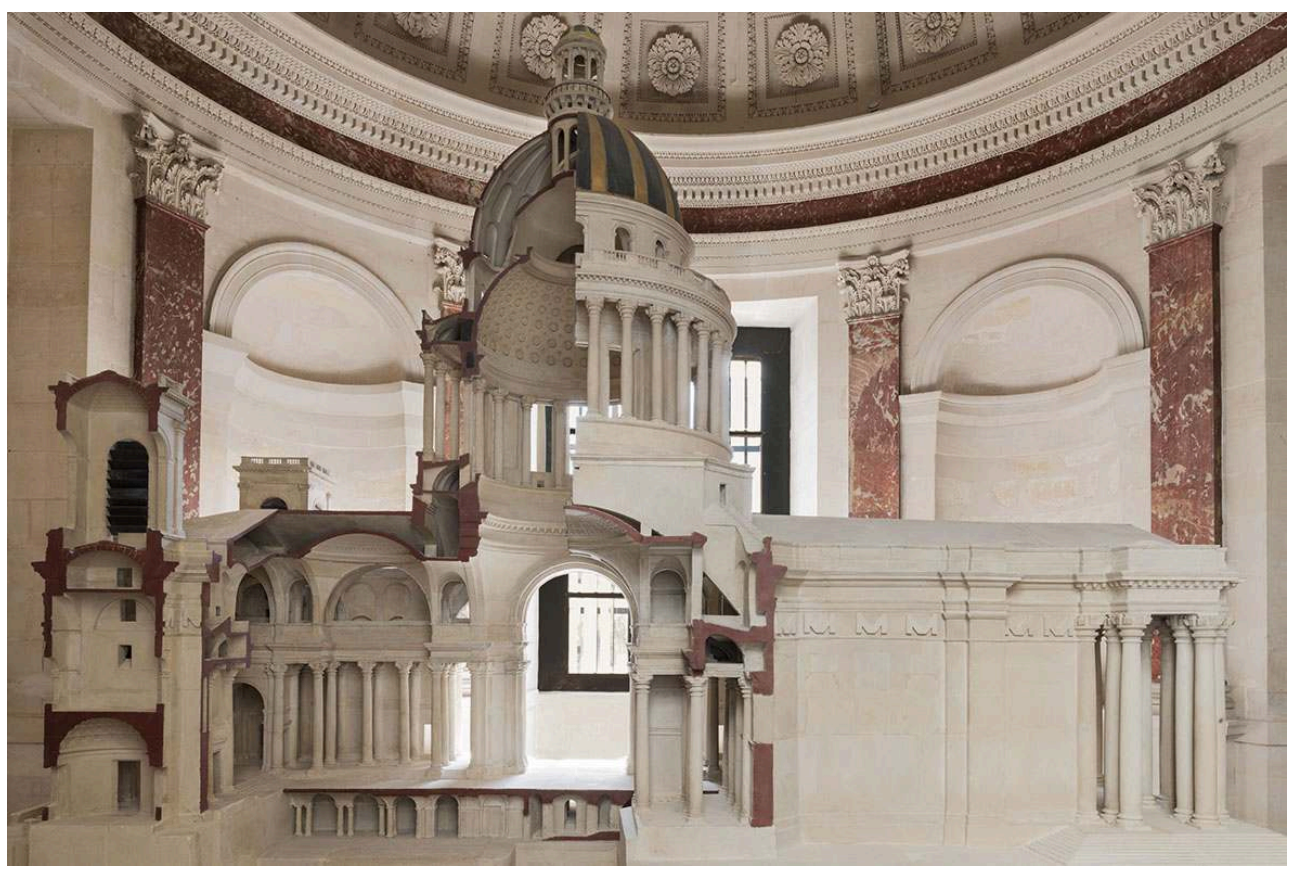

Maquette en pierre et plâtre attribuée à Jean-Baptiste Rondelet.

Phot. Benjamin Gavaudo. (C) Benjamin Gavaudo / Centre des monuments nationaux, 2017.

Entreposée au XIX ${ }^{e}$ siècle dans une salle de la crypte, la maquette fut installée en 1981 à la vue du public dans l'ancienne salle des évêques, au pied de la tour nord-est, de façon à pouvoir tourner autour ${ }^{23}$. Ce remarquable modèle figure parmi les quelques œuvres de cette période conservées en l'état avec les tombeaux de Voltaire et de Rousseau dont les cendres furent respectivement transférées en 1791 et 1794 . Si leur implantation a connu quelques péripéties ${ }^{24}$, les tombeaux des philosophes témoignent d'une inspiration antique qui associe un vocabulaire allégorique à un traitement polychrome du bois peint en faux marbre.

\section{Une succession d'affectations, des ensembles fragmentés}

Les périodes suivantes furent marquées par un va-et-vient permanent entre une destination civique et religieuse. Napoléon $\mathrm{I}^{\mathrm{er}}$ rendit le Panthéon au culte mais conserva un usage funéraire dans la crypte. Les inhumations se poursuivirent à un rythme soutenu pour honorer les dignitaires de l'Empire. Le tombeau du maréchal Lannes (1769-1809), à l'imitation des sarcophages antiques, figure parmi les réalisations de ces événements solennels qui ont été conservées. L'affirmation de cette orientation commémorative s'accompagna de commandes de monuments votifs. Dans ce contexte, trois fragments en bas-relief destinés au soubassement du mausolée du général Charles Victoire Emmanuel Leclerc ${ }^{25}$ ont été identifiés. L'ensemble est composé d'un plâtre original avec son négatif en creux ainsi que d'un bas-relief en marbre. Ces réalisations sont attribuées à Jean-Guillaume Moitte (1746-1810), disparu avant d'achever le monument, qui fut alors confié à François-Frédéric Lemot (1771-1827) ${ }^{26}$. Afin d'inscrire le régime dans la continuité de la Révolution, une sculpture en marbre de Voltaire fut commandée en 1806 à Jean-Antoine Houdon (1741-1828) afin d'être placée devant son tombeau (fig. 13). 
Figure 13

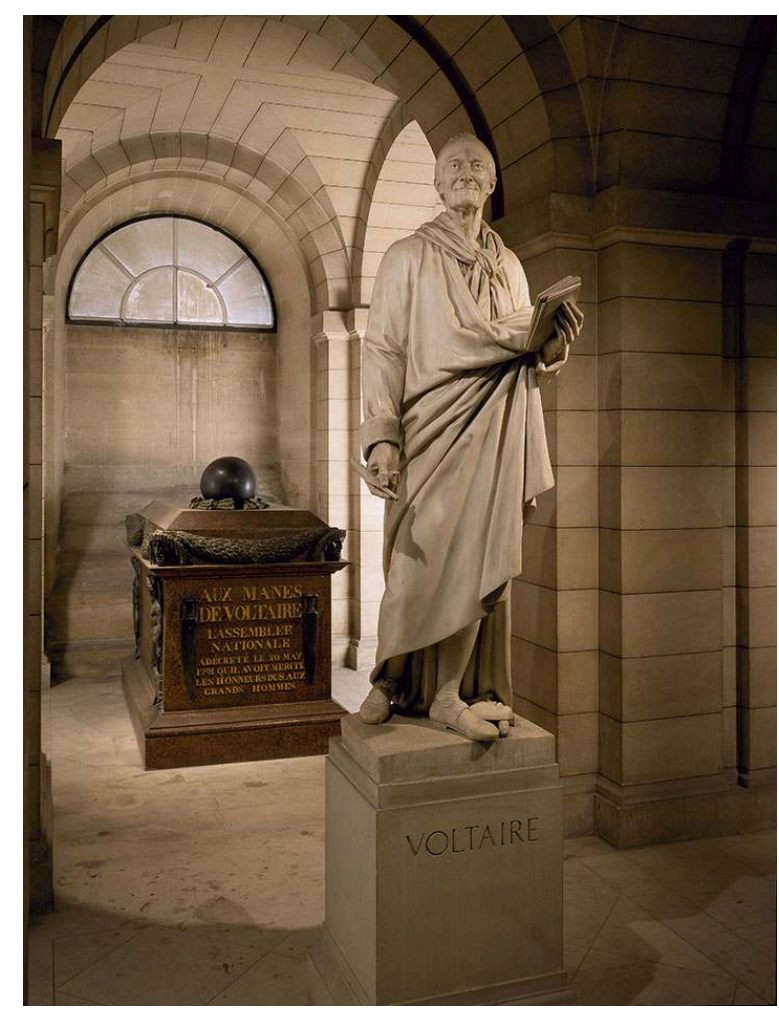

Tombeau de Voltaire et sculpture en marbre de Houdon, 1806-1812.

Phot. Caroline Rose. (c) Caroline Rose / Centre des monuments nationaux, 1995.

31 Avec le retour des Bourbons au pouvoir, le Panthéon redevient église dans sa totalité en 1816. L'architecte Louis-Pierre Baltard (1764-1846), qui prend la suite de Rondelet, nourrit l'ambition d'en faire une des principales églises du royaume. Les projets sont à peine achevés et les premières réalisations livrées que la révolution de 1830 affirme à nouveau la vocation civique du Panthéon. Baltard n'a que le temps de réaliser un mobilier cultuel dont il subsiste les aménagements intérieurs de la sacristie et une table calorifère en bronze doré à plateau de marbre (voir fig. 6). Cette pièce étonnante offre un condensé d'inspiration antique dans le traitement tripode du piétement à pattes de lion et emprunte à la Renaissance le choix du vocabulaire ornemental des rinceaux en bas-relief. La Restauration a pu mener à bien l'achèvement du décor peint de la coupole d'Antoine-Jean Gros (1771-1835), inauguré par Charles X en 1824, après un changement notoire de l'iconographie pour glorifier les figures monarchiques du royaume ${ }^{27}$.

Le nouveau régime modifie en profondeur l'usage du monument et s'engage dans des transformations ciblées du décor architectural. Le message délivré qui figure sur le fronton constitue le premier grand chantier de la monarchie de Juillet, qui rétablit l'inscription de 1791 du marquis Emmanuel de Pastoret, « Aux grands hommes la patrie reconnaissante ", et confie dès 1830 la commande du relief à David d'Angers (1788-1856), achevé en 1837. Les œuvres conservées pour cette période sont rares, seuls trois bas-reliefs en plâtre à moitié d'exécution relatifs au décor du péristyle se trouvent encore dans le monument. Il s'agit des modèles commandés à Charles-François Nanteuil-Lebœuf (1792-1865) pour les trois panneaux centraux : La Justice protégeant l'Innocence, Les Sciences et les Arts déposant les productions de leur génie sur l'autel de la Patrie 
(fig. 14) et Cérès enseignant l'agriculture à Triptolème, en remplacement des reliefs de l'époque révolutionnaire. On peut encore les voir aujourd'huii ${ }^{28}$. À l'intérieur de l'édifice, la commande des peintures des pendentifs à François Gérard (1770-1837), toujours en place, parachève le décor du dôme ${ }^{29}$.

Figure 14

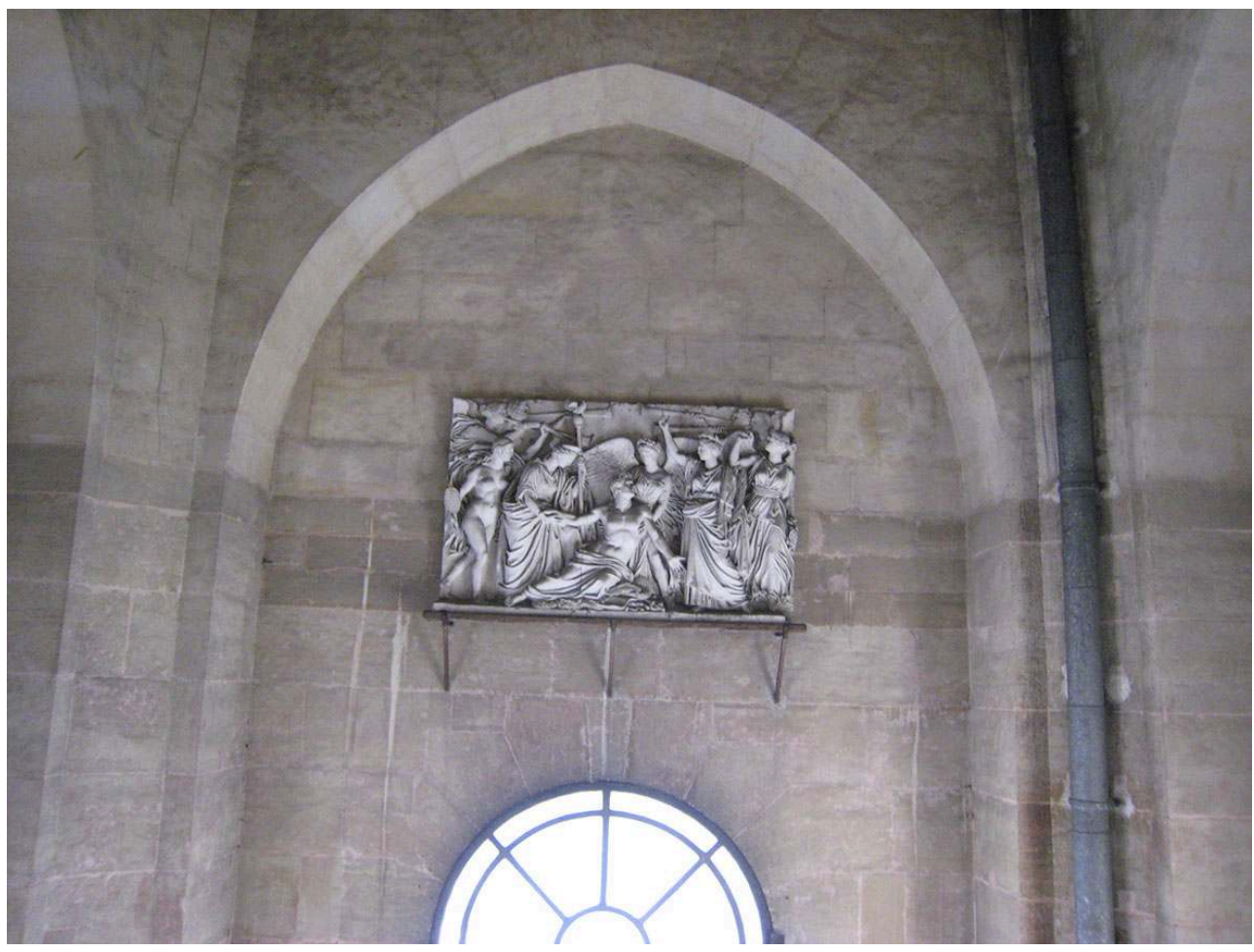

Charles-François Nanteuil-Lebœuf, Les Sciences et les Arts déposant les productions de leur génie sur l'autel de la Patrie, relief en plâtre, vers 1831-1834.

Phot. Élisabeth Portet. @ Élisabeth Portet / Centre des monuments nationaux, 2008.

La Révolution de 1848 et la brièveté de la Seconde République ont pour conséquence l'abandon des projets engagés pour le Panthéon. Le grand cycle de peintures sur l'histoire de l'humanité et son évolution morale par Paul Chenavard (1807-1895) est finalement resté inachevé. Seules les esquisses préparatoires conservées ${ }^{30}$ et présentées à l'Exposition universelle de 1855 témoignent de cette entreprise philosophique.

Le Second Empire, en revanche, a le temps de clôturer les différents chantiers entrepris en faveur d'une nouvelle destination de l'édifice, rendu au culte catholique.

Devenue basilique nationale en 1853, Sainte-Geneviève bénéficie de nouveaux aménagements intérieurs par l'architecte Simon-Claude Constant-Dufeux (1801-1870) qui réalise l'ensemble du mobilier liturgique : maître-autel, stalles, barrières de chœur et plusieurs autels. Certains biens subsistent à l'état fragmentaire dans les collections $\mathrm{du}$ Panthéon. Au moins trois parties du maitre-autel réalisé pour la cérémonie d'inauguration de l'église, lors de la translation des reliques de sainte Geneviève, le 21 janvier 1853, ont été identifiées. Ces éléments repérés permettent de reconstituer l'autel, en plâtre peint et métal doré, composé de la figure de sainte Geneviève au centre de quatre colonnes surmontées d'un entablement. Ce dernier sert de base à quatre figures supportant sur leurs mains élevées un petit monument quadrangulaire destiné à abriter la châsse en vermeil. Appartenant à cet ensemble lacunaire, les parties 
démontées du chemin de croix de l'atelier Cotelle ont été retrouvées dans les combles et rassemblées. Parmi les découvertes de l'inventaire figure une bannière de procession en satin de soie brodée à l'effigie de la Vierge et au chiffre de sainte Geneviève (fig. 15) probablement inspirée de la gravure de Dominique Sornique (1708-1756) gardant le souvenir de l'Immaculée Conception réalisée par Edme Bouchardon pour l'église SaintSulpice. Datée de 1852, elle avait été oubliée dans un placard de la sacristie et miraculeusement conservée.

\section{Figure 15}

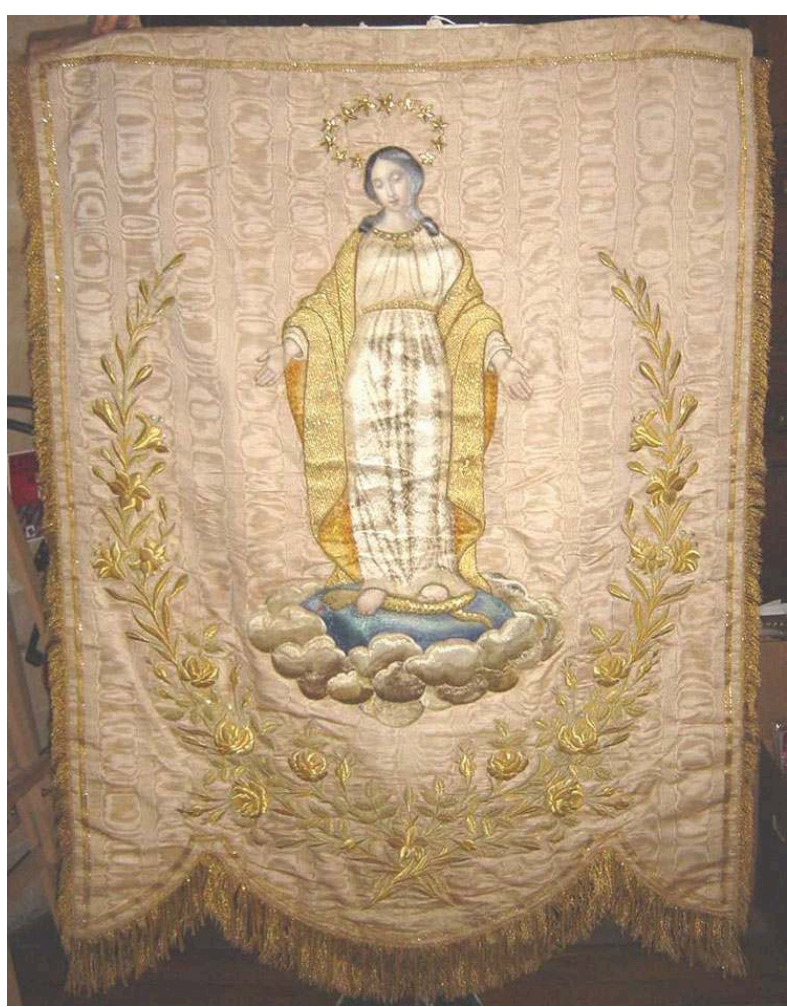

Bannière de procession en satin de soie, 1852

Phot. Ludovic Mathiez. (c) Ludovic Mathiez / Centre des monuments nationaux, 2005.

Hormis ces quelques exemples, les œuvres cultuelles du Panthéon connurent une destinée malheureuse en raison des nouveaux usages de l'édifice après 1885. Si les orgues de Cavaillé-Coll furent transférés cette année-là dans l'église Notre-Dame du Val-de-Grâce, ce qui a permis leur préservation, la plupart du mobilier cultuel fut dispersé, vendu (prie-Dieu, chaises) ou fondu (luminaires en bronze) sous l'Occupation.

\section{Les derniers revirements : entre grands programmes décoratifs et hommages funèbres}

La vocation religieuse du Panthéon fut confirmée sous la Troisième République durant la présidence du maréchal Patrice de Mac Mahon (1808-1893) entre 1873 et 1879 par les tenants de l'Ordre moral afin de signifier la reconquête religieuse de la société face au radicalisme républicain.

Dans cette perspective, Philippe de Chennevières, directeur des Beaux-Arts, et l'abbé Claude Bonnefoy, doyen de la basilique, définirent en 1874 un programme décoratif à la 
gloire de sainte Geneviève, célébrant à la fois l'histoire de France et le catholicisme. Les murs des quatre nefs, jusqu'alors dépourvus de décors, accueillirent progressivement les compositions monumentales exécutées sur toile marouflée par douze artistes sélectionnés. Les commandes s'échelonnèrent sur près de vingt ans, affirmant les fondements catholiques et monarchiques de la France dans des scènes peuplées de nombreux personnages historiques et royaux. Ces toiles, très bien documentées, ont fait l'objet de nombreuses études ${ }^{31}$ et ne nécessitent pas qu'on leur consacre ici un développement particulier.

Ce programme comprend aussi la réalisation de sculptures et de tapisseries. Si les statues du Christ, de la Vierge et de douze saints furent retirées en 1885, lorsque l'église devint définitivement Panthéon ${ }^{32}$, les deux tapisseries (fig. 16) exécutées dans ce contexte restèrent à leur emplacement d'origine, correspondant à l'arrière des autels de la Vierge et de sainte Geneviève. Les cartons des tentures murales furent exécutés par Charles-Joseph Lameire (1832-1910) puis tissés aux Gobelins selon la technique du point noué de la Savonnerie qui est exclusivement employée dans le cadre de cette commande en même temps que les tentures décoratives de l'Élysée et de la Bibliothèque nationale. Achevées en 1884, les tapisseries furent aussitôt présentées au public lors de l'exposition des manufactures nationales au palais de l'Industrie, sur les Champs-Élysées, comme exemple du savoir-faire français, avant de regagner le Panthéon.

Figure 16
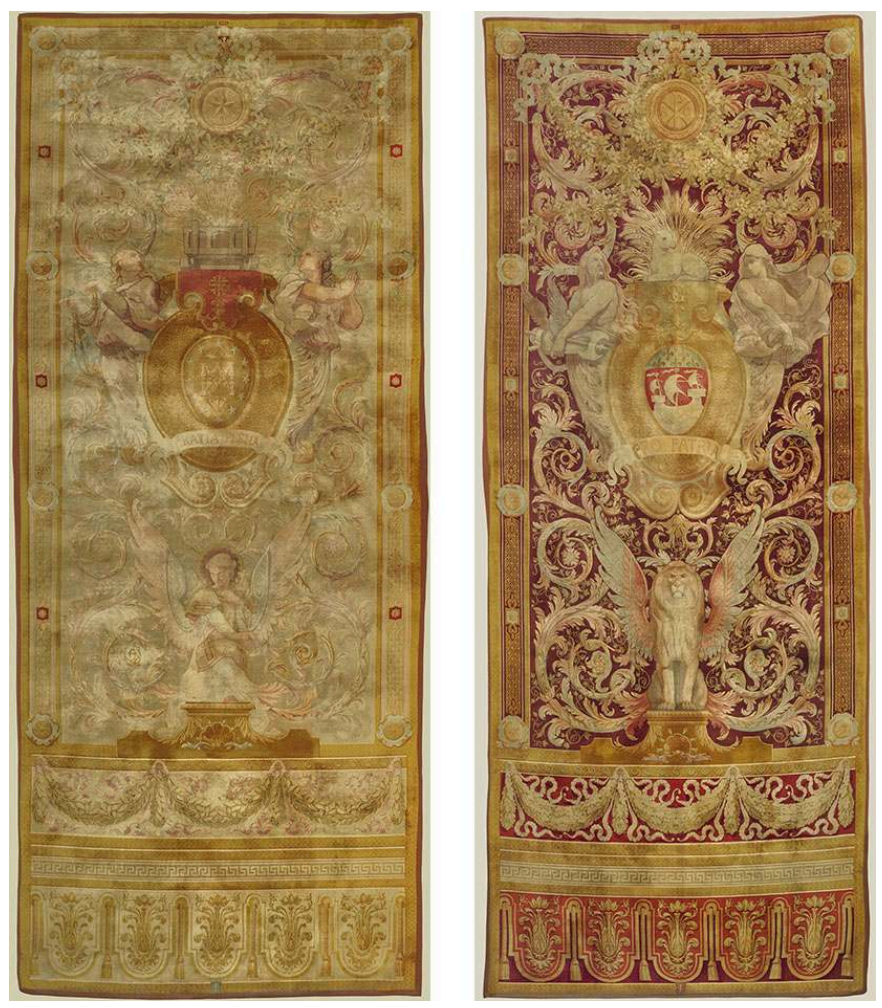

Tapisseries de la chapelle de la sainte Vierge et de la chapelle de sainte Geneviève, d'après CharlesJoseph Lameire, tissées aux Gobelins entre 1875 et 1884.

Phot. Hervé Lewandowski. @ C Hervé Lewandowski / Centre des monuments nationaux, 2017. 
Au lendemain du décès de Victor Hugo, le 22 mai 1885, le décret de Jules Grévy rendit à l'édifice sa destination républicaine, qui ne lui est plus retirée. Le programme décoratif initié en 1874 est complété par une nouvelle campagne de sculptures commémoratives qui consacre les sujets révolutionnaires, les thèmes allégoriques et l'effigie des grands hommes de l'histoire de France. Les groupes sculptés aujourd'hui visibles par le public dans le chœur et les quatre nefs appartiennent à cette série de commandes lancée par Gustave Larroumet (1852-1903), alors directeur des Beaux-Arts. L'installation de ces sculptures monumentales se fait au détriment des œuvres en place qui furent démontées en raison de leur inadéquation au nouveau programme projeté.

41 Les nouvelles orientations qui consacrent le Panthéon comme le lieu des célébrations nationales à la mémoire des grands hommes permettent de donner aux funérailles un éclat spectaculaire. Si les décors funèbres dressés à l'occasion des cérémonies n'ont pas été conservés, les ornements funéraires déposés l'ont été en partie. Les caveaux de la crypte offrent un aperçu des hommages mortuaires réalisés dans des styles variés et faisant preuve d'une grande richesse dans leurs matériaux. Le recensement de cet ensemble largement méconnu a mis en lumière l'identité des donateurs et la popularité des destinataires.

Sur les 75 ornements recensés, seules quelques couronnes mortuaires appartiennent à une production en série: la grande majorité d'entre elles présentent en effet un traitement original, dû à des commandes spéciales qui se plient aux volontés du commanditaire en révélant sa distinction ou sa position sociale.

Les couronnes déposées sur le caveau du président de la République Sadi Carnot $^{33}$ (1837-1894) font partie des réalisations les plus soignées. Deux exemples illustrent ce raffinement. Le premier est constitué par la grande couronne offerte par Ménélik II, roi d'Éthiopie, composée de feuilles de laurier et de chêne en zinc peint en vert, de velours noir, de galons dorés ainsi que d'un voile de crêpe de soie. Le deuxième est représenté par la plaque en laiton doré ornée d'un blason en cuivre émaillé champlevé réalisée par l'orfèvre Lucien Falize (1839-1897) à la demande de Nicolas II, tsar de Russie (fig. 17). 
Figure 17

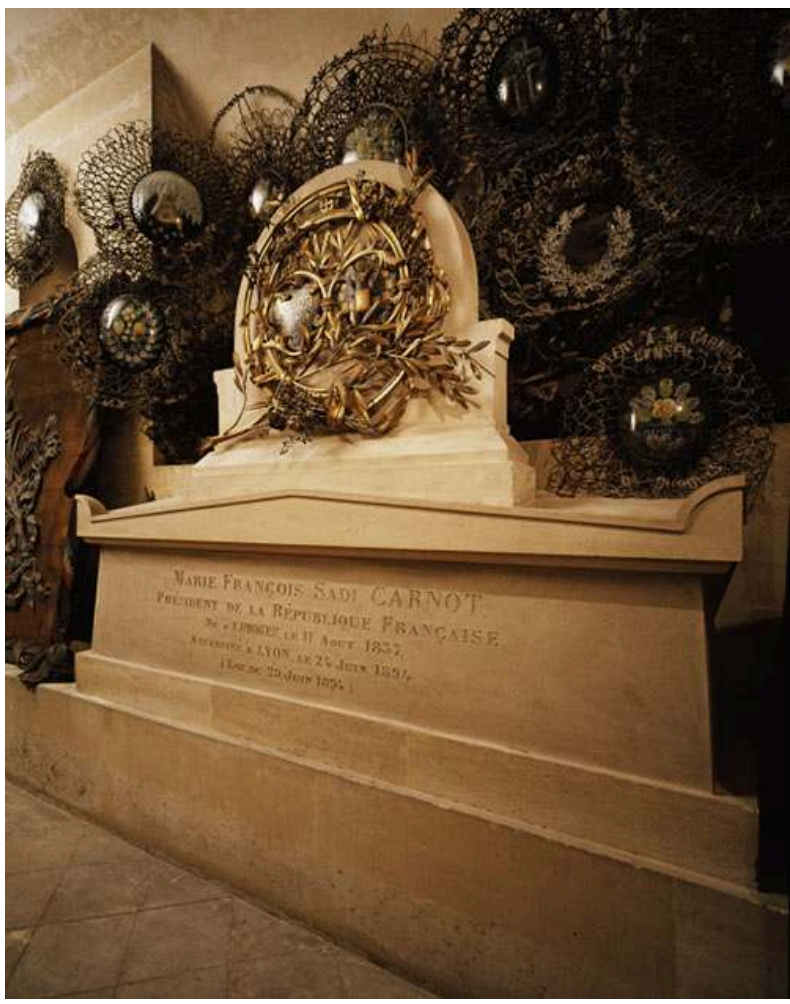

Ornements funèbres sur le caveau de Sadi Carnot.

Phot. Caroline Rose. (c) Caroline Rose / Centre des monuments nationaux, 1995.

\section{Perspectives de mises en valeur}

La clôture de l'inventaire permet de connaître l'intégralité des œuvres conservées dans le monument. Le premier constat qui en ressort est le faible nombre d'objets présentés au public, avec moins d'un tiers de la collection exposée. Ce chiffre s'explique par la quantité de décors déposés mais aussi par l'état de la recherche qui continue de progresser au fur et à mesure des découvertes. La prise en compte à l'inventaire d'ensembles jusqu'alors peu étudiés offre de nouvelles perspectives dans le traitement de leur conservation matérielle, de leur restauration et de leur diffusion.

\section{Sauvegarde matérielle et présentation des collections}

Un des principaux enjeux de l'inventaire et du récolement des collections est de s'inscrire dans la continuité de la chaîne patrimoniale pour aboutir à la valorisation auprès $d u$ public. L'étape indispensable du recensement permet d'initier très tôt le processus de conservation avec le regroupement des collections par typologies ou par matériaux. Dès leur entrée dans l'inventaire, les collections d'objets liturgiques du Panthéon ont été rassemblées dans les salles hautes, tandis que les œuvres lapidaires faisaient l'objet d'un rangement soigné par une équipe de transporteurs spécialisés.

En 2010, le récolement des décors d'architecture en plâtre, dont les ensembles avaient déjà été regroupés lors de leur inventaire en 1991, a montré la nécessité de poursuivre 
cette entreprise, inachevée faute de moyens logistiques. Entassés et empoussiérés, les plâtres exigeaient une prise en charge spécifique pour leur conservation.

Dans le cadre de l'étude transversale menée en 2013-2014 par le pôle de la conservation préventive sur les collections de plâtres de neuf monuments de l'établissement ${ }^{34}$ et des réflexions engagées au sein du réseau des "gypsothèques", un chantier des collections a démarré en 2017 sur ces décors (fig. 18). Les plâtres ont bénéficié d'une importante campagne de conservation qui vise à les dépoussiérer et à reconstituer les ensembles dispersés. Le regroupement des fragments suivant la chronologie des commandes est un objectif à atteindre en tenant compte des contraintes techniques de régie des œuvres et d'optimisation des espaces de réserves dans les tours nord-est et sud-est.

Figure 18

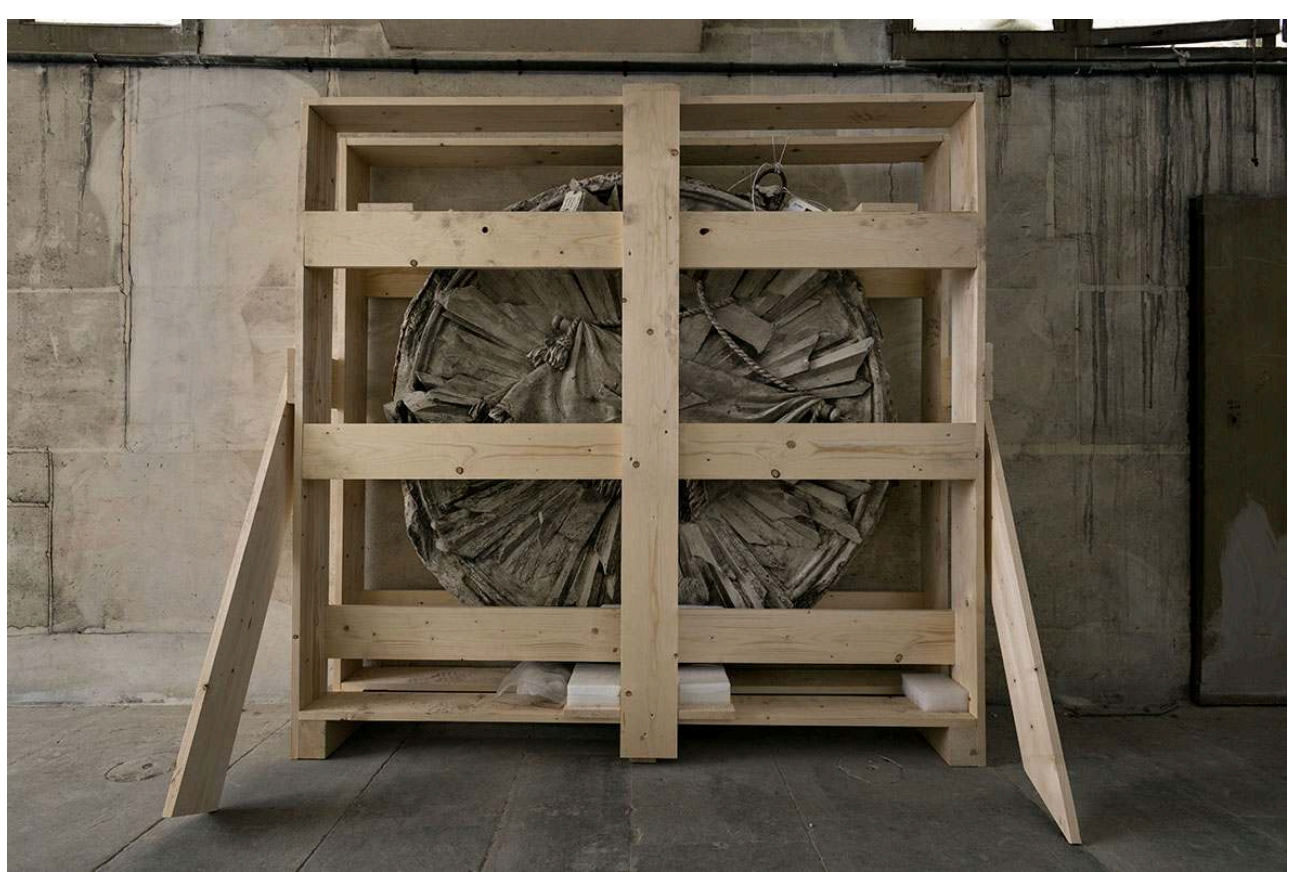

Chantier des collections des décors d'architecture en plâtre : mise en caisse du médaillon avec le labarum de Constantin.

Phot. Benjamin Gavaudo. (C) Benjamin Gavaudo / Centre des monuments nationaux, 2017.

Cette opération est complétée en 2018 par le marquage des plâtres qui sont conservés sur de nouveaux rayonnages après traitement afin d'assurer leur préservation matérielle et leur diffusion. Face aux possibilités restreintes d'accrochage dans le monument et compte tenu de l'intérêt historique de la collection, l'aménagement de deux salles de réserves permet de les rendre accessibles aux visiteurs et aux chercheurs, sur demande.

Pour ces ensembles qui ont une valeur de témoignage irremplaçable, la logique de sauvegarde in situ à travers la configuration de réserves "visitables " peut être envisagée.

Pour d'autres, la logique de présentation historique est un axe intéressant à approfondir. À la suite de l'inventaire des ornements funéraires de la crypte, le besoin urgent d'une intervention en conservation-restauration a été défini dans la perspective de leur mise en valeur. Au-delà de la simple conservation matérielle, l'enjeu était double : il s'agissait de pouvoir étudier les matériaux constitutifs des décors funèbres et 
d'envisager de nouvelles formes de présentation après repérage des dédicaces. Le chantier effectué en $2015^{35}$ a ainsi mis l'accent sur l'intérêt de poursuivre la réfection de l'accrochage des ornements funéraires dans les caveaux. Cette présentation suit fidèlement les dispositions d'origine qui sont bien connues par les photographies d'époque.

En dernier recours, la logique de présentation muséographique peut s'appliquer mais elle connaît cependant des difficultés à s'inscrire dans la durée. En 1990, à la suite de l'exposition organisée sur le bicentenaire de la Révolution, la galerie sud de la crypte a bénéficié d'un aménagement scénographique (fig. 19) pour retracer les grandes lignes de l'histoire du Panthéon ${ }^{36}$. À cette occasion, quelques fragments en pierre et en plâtre découverts dans les parties hautes ont été sélectionnés pour une présentation au public. Cet accrochage doit aujourd'hui être revu pour s'adapter aux nouvelles normes et montre la difficulté d'investir un espace pérenne au sein du monument.

Figure 19

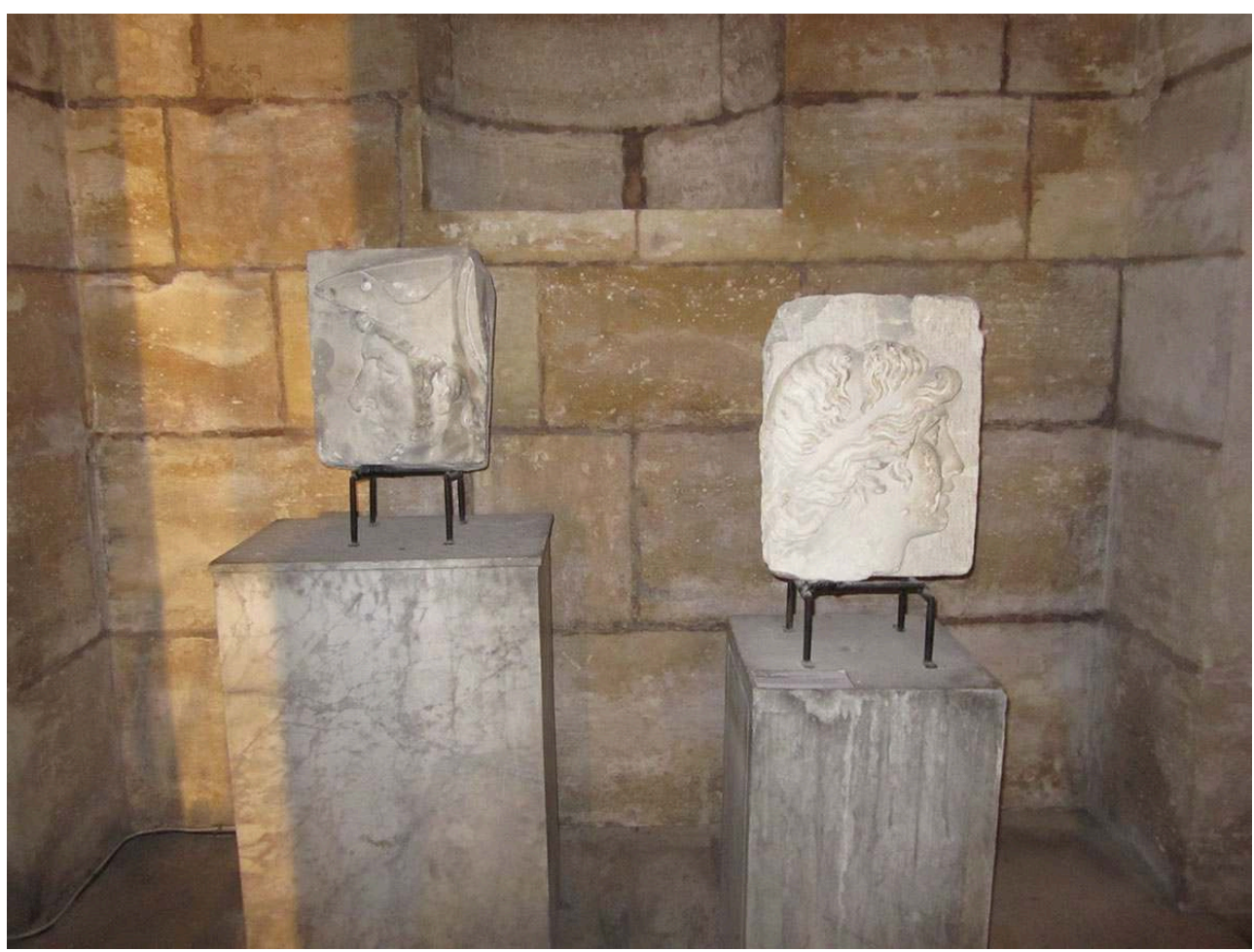

Exposition d'œuvres lapidaires dans la galerie sud de la crypte.

Phot. Élisabeth Portet. (c) Élisabeth Portet / Centre des monuments nationaux, 2009.

Ces tentatives de mise en valeur des collections dans le parcours de visite peuvent être complétées par une actualisation ponctuelle de l'accrochage qui permettrait d'exposer les dernières acquisitions de l'établissement comme l'esquisse très aboutie peinte par Jules Lenepveu (1819-1898) pour le cycle de Jeanne d'Arc ou les aquarelles de PierreFrançois-Léonard Fontaine (1762-1853) tirées de l'album d'Alexandre $\mathrm{I}^{\mathrm{er}}$ de Russie (fig. 20). Ces œuvres inédites pourraient faire l'objet d'une exposition-dossier en vue de leur présentation au public. 
Figure 20

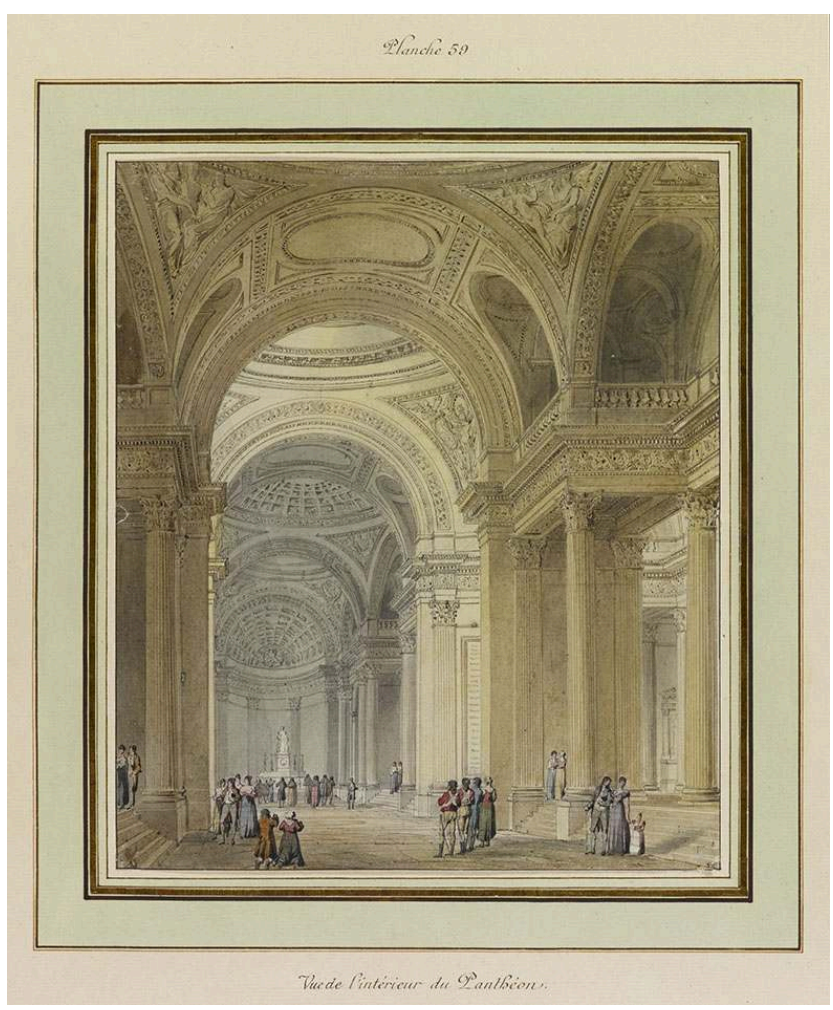

Fontaine, Vue de l'intérieur du Panthéon, aquarelle, planche 59 de l'album de l'empereur de Russie, 1810, acquis par le Centre des monuments nationaux (pour le compte de l'État) en 2012.

Phot. Philippe Berthé. (c) Reproduction Philippe Berthé / Centre des monuments nationaux, 2012.

L'éclairage scientifique apporté par l'organisation d'une exposition temporaire sur un sujet défini reste un bon vecteur de diffusion de la connaissance. C'est d'ailleurs dans le cadre de l'exposition Soufflot organisée en 2013 au Panthéon qu'un premier essai de présentation des décors d'architecture en plâtre a été mis en œuvre. Trois médaillons et un bas-relief ont été sortis des réserves ${ }^{37}$ après restauration pour permettre aux visiteurs d'appréhender une partie des décors déposés de la première église SainteGeneviève.

\section{Diffusion et médiation}

Les quelques cas évoqués soulignent l'ampleur de la tâche et la difficulté d'exposer de façon permanente des œuvres qui ont perdu leur valeur d'usage. Alors que la plupart des décors sont conservés dans les réserves et ne sont plus en adéquation avec la vocation actuelle du monument, comment, dans ces conditions, envisager leur présentation? Un début de réponse se trouve dans le recours aux technologies numériques et leurs nouvelles perspectives pour l'identification, la connaissance et la médiation du patrimoine. L'apport du numérique à la diffusion des objets patrimoniaux, via les bases de données, permet de les diffuser. Dans cette perspective et en attendant un portail de ses collections en ligne, le Centre des monuments nationaux contribue à l'alimentation de la base de données Palissy du ministère de la Culture en reversant les inventaires validés. Les informations collectées lors de l'inventaire sont ainsi mises à la disposition du public. 
55 L'installation de nouvelles bornes numériques, en 2017, accompagne de façon pédagogique l'offre de visite en proposant des explications interactives aux visiteurs (fig. 21). Au même moment, la maquette en pierre de Rondelet a été restaurée et mise en valeur par une nouvelle scénographie. Deux pupitres tactiles fixés sur les mises à distance permettent au public de commander des points lumineux qui soulignent les détails architecturaux du monument en donnant une description détaillée de la partie choisie ${ }^{38}$.

Figure 21

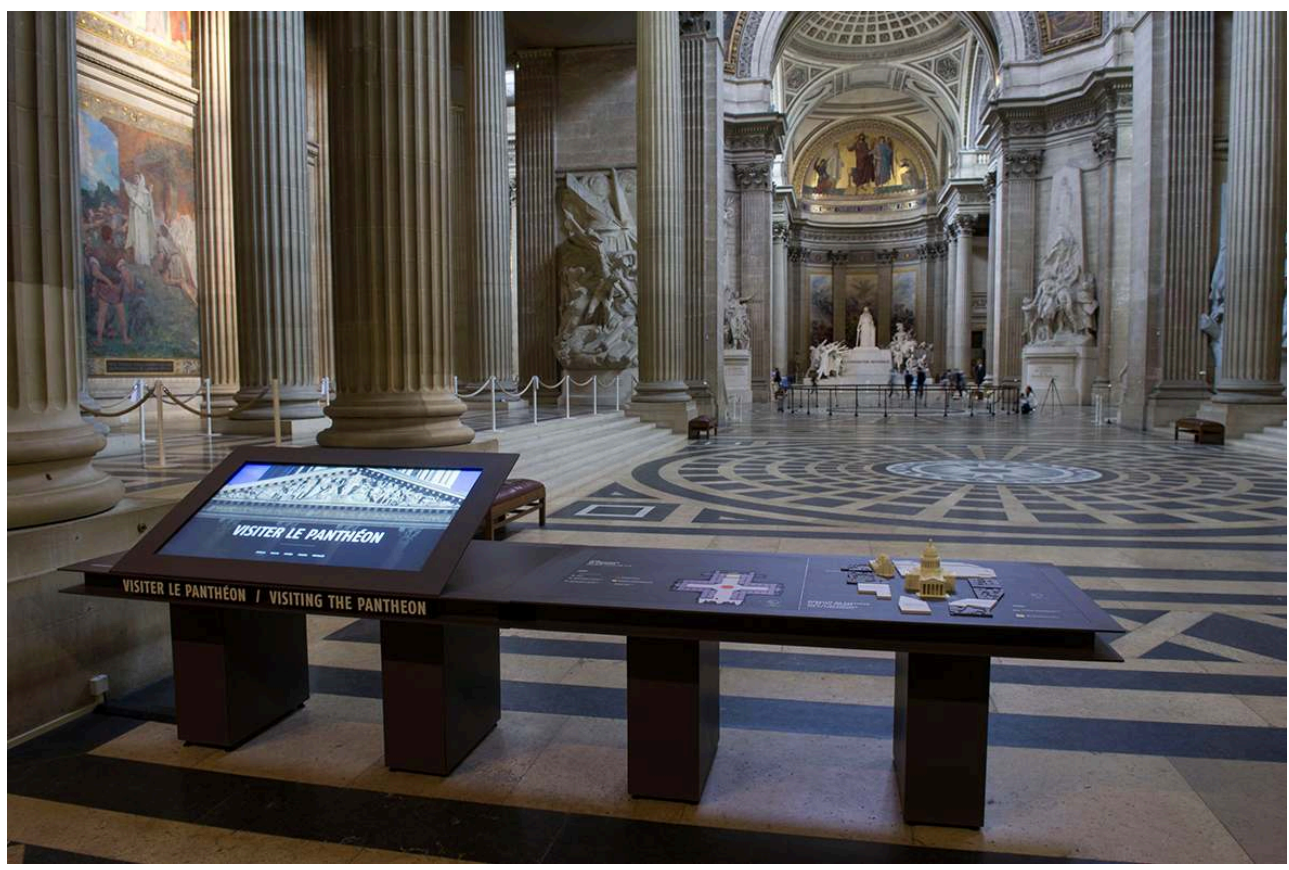

Nouveaux supports de médiation au Panthéon.

Phot. Didier Plowy. ㄷ Didier Plowy / Centre des monuments nationaux, 2017.

Ces initiatives pourraient être complétées par les récents dispositifs d'applications immersives de visite en 3D faisant appel à la réalité augmentée, déjà développés dans certains monuments de l'établissement ${ }^{39}$, qui trouveraient au Panthéon un formidable écho. En replaçant chaque fragment de décor dans son contexte historique, ils offriraient la possibilité de voir les collections des réserves et d'en comprendre la destination initiale.

Les collections liturgiques trouveraient notamment une place de choix pour se projeter, par exemple, dans les aménagements du Second Empire. Ce sujet mérite des recherches scientifiques complémentaires pour travailler sur l'identification des objets mobiliers dispersés qui ont été préservés. Une partie a été localisée dans les réserves du Mobilier national et une étude comparative de ces ensembles permettrait de préciser les provenances et d'affiner les attributions.

58 Au-delà de l'apport du numérique, le recours à la restitution est une solution pour appréhender certaines expériences essentielles à la compréhension du monument. C'est le cas du pendule de Léon Foucault (1819-1868) qui fut installé à deux reprises au Panthéon, en 1851 puis en 1902. L'oscillation légère de l'instrument au sol, suspendu à un câble d'acier sous le dôme de l'édifice, démontre les mouvements de rotation de la 
Terre. Afin d'évoquer ces épisodes scientifiques célèbres, la sphère actuellement présentée au public est une reconstitution de 1995 faite à partir de l'original en laiton conservé au musée des Arts et Métiers ${ }^{40}$.

S'il reste beaucoup à faire pour approfondir les connaissances et améliorer les conditions de conservation des collections dans le monument, la démarche engagée devrait se poursuivre dans les prochaines années pour renouveler l'intérêt des visiteurs et préserver le Panthéon comme « lieu vivant de la mémoire nationale »"

\section{NOTES}

1. - QUINET, Edgar. Les Églises de Paris. Le Panthéon. Paris : C. Marpon et É. Flammarion, 1883, p. 154-155.

2. - L'église est construite sur une partie des terrains de l'ensemble médiéval des chanoines génovéfains qui menaçait ruine, afin de glorifier la monarchie à travers la figure de la sainte patronne de Paris.

3. - Parution au Journal officiel du 26 mai 1885. À l'article 2, il est précisé que la translation des restes des grands hommes au Panthéon sera ordonnée par décret du président de la République.

4. - BÉLAVAL, Philippe. Pour faire entrer le peuple au Panthéon. Rapport à Monsieur le Président de la République. Octobre 2013. Mission confiée à M. Philippe Bélaval, président du Centre des monuments nationaux, pour étudier la manière de renforcer le rôle du Panthéon dans la diffusion des valeurs universelles de la France.

5. - Cérémonie de translation des cendres de quatre personnalités de la Résistance, Geneviève de Gaulle-Anthonioz, Pierre Brossolette, Germaine Tillion et Jean Zay, qui a eu lieu le 27 mai 2015.

6. - Cérémonie d'inhumation de Simone Veil et de son époux, Antoine Veil, qui s'est tenue le $1^{\text {er }}$ juillet 2018.

7. - Pour en savoir plus sur les méthodes d'inventaire et de récolement des collections du CMN, voir le site: https://www.monuments-nationaux.fr/Espace-Decouvertes/Inventaire-etrecolement-des-collections [consulté le 20/02/2018].

8. - Voir le site : https://tapisseries.monuments-nationaux.fr [consulté le 29/01/2018].

9. - CHENNEVIÈRES, Philippe de. «Le Panthéon. Ancienne église de Sainte-Geneviève ». Dans Inventaire général des richesses d'art de la France. Paris. Monuments civils (église Sainte-Geneviève). Paris : Plon, 1889, vol. 2, p. 331-349.

10. - Le Panthéon. Symbole des révolutions. De l'église de la Nation au temple des grands hommes. Cat. exp., Paris, hôtel de Sully, 31 mai-30 juillet 1989; Montréal, Centre canadien d'architecture, 19 septembre-15 novembre 1989. Paris/Montréal : CNMH-Picard/Centre canadien d'architecture, 1989.

11. - Je tiens à remercier Antonine Sergent et Lucas Rolls qui m'ont accompagnée sur place pendant plusieurs semaines pour effectuer le récolement des plâtres ainsi que l'inventaire des ornements funèbres. J'exprime toute ma gratitude envers Francine Pin, régisseur des œuvres, pour son soutien logistique lors de l'opération de l'inventaire du lapidaire, et Ludovic Mathiez, chef de pôle, pour son aide lors de l'inventaire des ornements liturgiques.

12. - VERDIER, Hélène (dir.). Système descriptif des objets mobiliers. Paris : Éditions du patrimoine, 1999 ; VERDIER, Hélène (dir.). Thesaurus des objets mobiliers. Paris : Éditions du patrimoine, 2001. 
13. - La base de données d'inventaire et de gestion des collections a été mise en place à partir de 2002 sur le logiciel Access 97 de Microsoft. Devenu obsolète, le système informatique a été remplacé en 2013 et a basculé vers le logiciel The Museum System (TMS) de la société Collections Information Technology (CIT).

14. - Voir, dans la base Palissy, notices: PM75001724 pour la maquette; PM75001725 pour le calorifère ; PM75002782 pour les plâtres. Voir dans la base Mérimée : notice PA00088420.

15. - La Commission de récolement des dépôts d'œuvres d'art (CRDOA) a été créée par un décret du 20 août 1996 pour vérifier la présence sur place et sur pièce d'œuvres appartenant à l'État déposées chez les dépositaires. Voir le site : http://www.culturecommunication.gouv.fr/Nousconnaitre/Organisation/Services-rattaches-a-la-ministre/Commission-de-recolement-desdepots-d-oeuvres-d-art/Presentation-de-la-CRDOA [consulté le 11/02/2018].

16. - Plâtre original du monument en bronze élevé par l'association des «Bleus de Bretagne » pour la ville de Quiberon et inauguré en 1902. Il en existe un autre exemplaire, à Versailles, devant l'hôtel de ville.

17. - En 1744 Louis XV, malade, forma le vœu de faire construire, s'il guérissait, une église dédiée à sainte Geneviève. La guérison étant advenue, l'architecte Soufflot fut choisi pour conduire le projet, financé par la loterie royale. La première pierre fut posée en 1764, la dernière en 1790 par Jean-Baptiste Rondelet, élève de Soufflot, mort en 1780.

18. - FRANCQUEVILLE, Anne, LAROCHE, Caroline. Rapport d'inventaire des modèles originaux en plâtre et fragments en pierre déposés dans le Panthéon. Août 1988-mai 1989.

19. - Nicolas-François Dupré, 1739-1787.

20. - COUSTOU, Guillaume. Mémoire des ouvrages de sculptures faites à la nouvelle église SainteGeneviève. Archives nationales 0/1, 1698-74.

21. - La décoration des voûtes des quatre nefs est remplacée par des figures allégoriques. Les représentations de l'Histoire, la Science, la Morale, l'Agriculture ou la Navigation et le Commerce constituent quelques exemples de ce nouveau décor.

22. - LEFEVRE, Daniel. Étude préalable à la restauration du dôme et du tambour. 2007, t. 1, p. 16.

23. - Ce transfert a posé rapidement un problème de répartition des charges au sol. Un étaiement de sécurité a été installé en 1982 dans la salle située en-dessous.

24. - Les tombeaux des deux philosophes furent discrètement déplacés en 1821 sous le portique du péristyle. Après les événements de juillet 1830 , ils retrouvèrent leur place dans l'aile est de la crypte. Ce transfert fut sans doute à l'origine des rumeurs accréditant l'hypothèse que les caveaux ne contenaient pas leurs dépouilles. L'ouverture des sarcophages, à trois reprises, démontra que ces rumeurs étaient sans fondement.

25. - 1772-1802, premier époux de Pauline Bonaparte.

26. - LEMAISTRE, Isabelle. «De sainte Geneviève au Panthéon: les différents programmes de sculpture, à la lumière des récentes découvertes ». Dans Le Panthéon. Symbole des révolutions. Op. cit ., p. 241-242. Longtemps considéré comme l'œuvre de Jean-Baptiste Stouf (1742-1826) et PhilippeLaurent Roland (1746-1816), Isabelle Lemaistre attribue l'achèvement du monument à Lemot.

27. - Débutée en 1811, la peinture de L'Apothéose de sainte Geneviève a été modifiée pour remplacer le couple impérial par les figures de Louis XVIII, de la duchesse d'Angoulême et du duc de Bordeaux.

28. - Entre 1815 et 1827 , les bas-reliefs révolutionnaires du péristyle furent déposés. Cinq nouveaux panneaux sur le thème de sainte Geneviève furent commandés pour les remplacer. Le jeune David d'Angers se vit confier la réalisation des reliefs centraux tandis que les panneaux latéraux étaient commandés à Raymond Gayrard (1807-1855) et Valcher. Les événements de 1830 laissent le travail inachevé. Les bas-reliefs d'Antoine-Denis Chaudet (1763-1810) et JacquesPhilippe Le Sueur (1757-1830) sont remis en place et les trois panneaux centraux sont confiés à Nanteuil. 
29. - Les pendentifs de Gérard commandés en 1831 illustrent des thèmes allégoriques universels : la Gloire, la Mort, la Justice et la Patrie.

30. - Une partie des esquisses préparatoires et des peintures en grisaille sont conservées au musée des Beaux-Arts de Lyon.

31. - MACÉ de LÉPINAY, François. Peintures et sculptures du Panthéon. Paris: Éditions du Patrimoine, 1997.

32. - Les sculptures de huit statues de saints furent transférées en 1885 au dépôt des marbres de Paris avant d'être installées en 1922 à la cathédrale d'Arras (Pas-de-Calais) où elles sont toujours présentées.

33. - Assassiné en 1894.

34. - RAGER, Geneviève. "Plans de sauvegarde et monuments nationaux : réflexion sur les priorités de conservation à propos des collections de moulages ", In Situ [En ligne], 28 | 2016, mis en ligne le 11 mars 2016, consulté le 19 juin 2018. URL : http://journals.openedition.org/insitu/ 12623 ; DOI : 10.4000/insitu.12623.

35. - Chantier de dépoussiérage et bilan sanitaire de la collection d'ornements funéraires mené en 2015 par le groupement Émile, Bayol, Bentouati, Detallante, Petit, Verwaerde et Zelinski.

36. - Scénographie conçue par Gida Ricci à partir des contenus synthétisés de l'exposition de 1989 sur le Panthéon présentée à Paris puis à Montréal.

37. - Dans le cadre de cette exposition, qui s'est tenue au Panthéon du 10 septembre au 23 novembre 2013, sous le commissariat d'Alexandre Gady, les plâtres suivants ont été exposés : Présentation du temple au roi Salomon, Isaac bénissant Jacob, médaillon au chiffre de Louis XVI, Portrait de Louis XVI et Sainte Geneviève rendant la vue à sa mère.

38. - NIEDZIOCHA, Christophe. «La restauration et la mise en valeur de la maquette de chantier : relier la forme à l'esprit du Panthéon». Dans Monumental, Semestriel 2. Paris : Éditions du Patrimoine, 2017, p. 68-69.

39. - Les nouvelles expériences de visite virtuelle en 3D ont été développées pour reconstituer l'abbaye de Cluny en 2010, à la villa Cavrois en 2015 pour projeter les visiteurs dans des intérieurs meublés et à la Conciergerie en 2016 avec l'HistoPad sur écran tactile.

40. - DELIGEORGES, Stéphane. Le Pendule de Foucault au Panthéon 1851-1902-1995 [1995]. Paris: Éditions du Patrimoine, 2009.

41. - OZOUF, Mona. « Le Panthéon, l'École normale des morts ». Dans NORA, Pierre (dir.). Les Lieux de mémoire. Paris : Gallimard, 1984-1992, t. 1, La République [1984], 1997, p. 155.

\section{RÉSUMÉS}

Le Centre des monuments nationaux (CMN) assure la gestion, la conservation et la diffusion des collections du Panthéon (Paris), qui ont à ce titre fait l'objet d'une campagne pluriannuelle d'inventaire et de récolement à partir de 2005. Les données collectées permettent de mieux connaître les ensembles mobiliers présents dans le monument ( 637 biens culturels répertoriés) et de programmer des interventions de conservation en vue de leur valorisation auprès de tous les publics. Les méthodes employées pour mener cette mission, achevée en 2016, et les premiers résultats de ce travail sont présentés dans cet article qui reconsidère également la perception archéologique et fragmentaire du corpus constitué. 
The Centre des monuments nationaux is responsible for the management, promotions and interpretation of the collections of the Panthéon, which have been the subject of an inventory campaign undertaken from 2005. The data collected gives us better information about the moveable heritage present in the monument (637 items listed) and allows for conservation interventions to be properly planned with a view to interpreting the objects for the public at large. The methods used to carry out this mission, which was completed in 2016, and the first results of the undertaking are presented in this article which also gives consideration to the archaeological and fragmentary perception of the corpus constituted.

\section{INDEX}

Mots-clés : Panthéon, église Sainte-Geneviève, Centre des monuments nationaux, inventaire, récolement, gypsothèque, moulages, lapidaire, peinture, tapisserie, décors funèbres, réserves, monuments historiques, base de données, régie, grands hommes

Keywords : Panthéon, Sainte-Geneviève church, Centre des monuments nationaux, inventory, plaster cast collection, lapidary, painting, tapestry, funereal decoration, reserve collections, historic monuments historique, data base, collection management, 'great men'

\section{AUTEUR}

\section{ÉLISABETH PORTET}

Chargée d'inventaire des collections, Direction de la conservation des monuments et des collections, Centre des monuments nationaux elisabeth.portet@monuments-nationaux.fr 